Document downloaded from:

http://hdl.handle.net/10251/79725

This paper must be cited as:

Defant, A.; Schwarting, U.; Sevilla Peris, P. (2014). Estimates for vector valued Dirichlet polynomials. Monatshefte fr Mathematik. 175(1):89-116. doi:10.1007/s00605-013-0600-4.

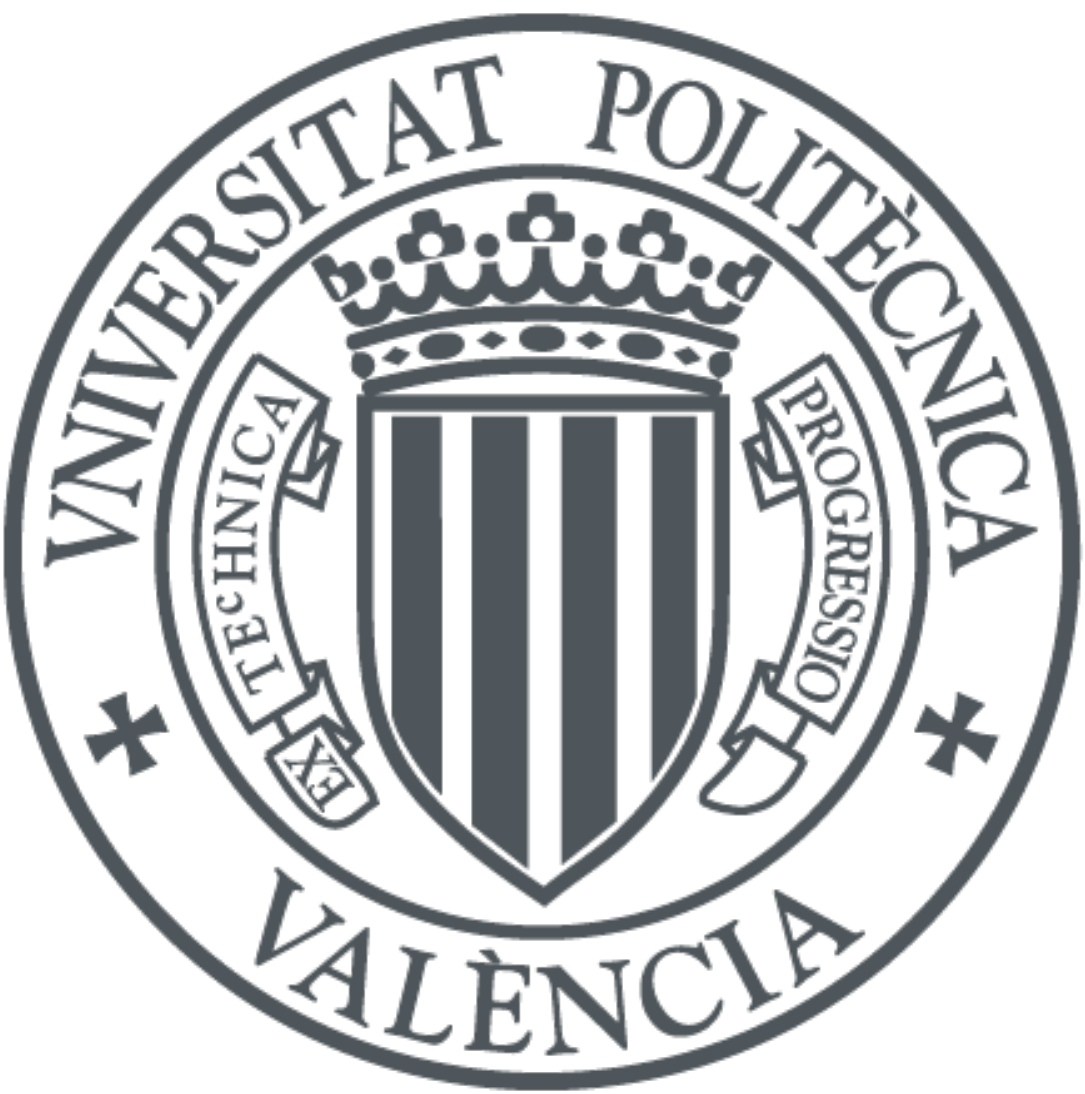

The final publication is available at

http://dx.doi.org/10.1007/s00605-013-0600-4

Copyright Springer Verlag (Germany)

Additional Information 


\title{
ESTIMATES FOR VECTOR VALUED DIRICHLET POLYNOMIALS
}

\author{
ANDREAS DEFANT, URSULA SCHWARTING, AND PABLO SEVILLA-PERIS
}

\begin{abstract}
We estimate the $\ell_{1}$-norm $\sum_{n=1}^{N}\left\|a_{n}\right\|$ of finite Dirichlet polynomials $\sum_{n=1}^{N} a_{n} n^{-s}$, $s \in \mathbb{C}$ with coefficients $a_{n}$ in a Banach space. Our estimates quantify several recent results on Bohr's strips of uniform but non absolute convergence of Dirichlet series in Banach spaces.
\end{abstract}

\section{INTRODUCTION}

It is a well known fact from the classical theory that each Dirichlet series $D=\sum_{n} a_{n} n^{-s}$ defines four significant abscissas: the infimum over all $\sigma$ such that in the halfplane $[\operatorname{Re}>\sigma]$ the Dirichlet series converges $\left(\sigma_{c}(D)\right)$, defines a bounded, holomorphic function $\left(\sigma_{b}(D)\right)$, converges uniformly $\left(\sigma_{u}(D)\right)$ or converges absolutely $\left(\sigma_{a}(D)\right)$.

H. Bohr showed in his fundamental Theorem [6, Satz I] that $\sigma_{b}(D)=\sigma_{u}(D)$, and his main interest was to determine the largest possible width of the band on which a Dirichlet series can converge uniformly but not absolutely; in other words, to find the precise value of

$$
S=\sup \sigma_{a}(D)-\sigma_{u}(D)
$$

where the supremum ranges over all possible Dirichlet series $D$. The now called BohrBohnenblust-Hille Theorem [4,5] shows that $S=\frac{1}{2}$.

In the last few years there has been a renewed interest in this result, and it has been looked at from different points of view. If we denote by $\mathcal{H}^{\infty}$ the Banach space of all Dirichlet series that define a bounded and holomorphic function on $[\mathrm{Re}>0]$ (together with the sup norm), then we can rewrite

$$
S=\sup _{D \in \mathcal{H}^{\infty}} \sigma_{a}(D)
$$

and by the Bohr-Bohnenblust-Hille Theorem every Dirichlet series in $\mathcal{H}^{\infty}$ converges absolutely on $\left[\operatorname{Re}=\frac{1}{2}+\varepsilon\right]$, and the value $\frac{1}{2}$ is optimal. A natural question then is to ask whether this even holds for $\varepsilon=0$; that is: is it true that for every Dirichlet series in $\mathcal{H}^{\infty}$ we have $\sum_{n} \frac{\left|a_{n}\right|}{n^{\frac{1}{2}}}<\infty$ ? Extending the work of Konyagin and Queffélec from [20] (see also [27]) Balasubramanian, Calado and Queffélec in [1, Theorem 1.1] gave a positive answer to this question. But they prove a lot more: there is a constant $C>0$ such that for every $\sum a_{n} n^{-s} \in \mathcal{H}^{\infty}$

$$
\sum_{n=1}^{\infty}\left|a_{n}\right| \frac{e^{C \sqrt{\log n \log \log n}}}{n^{\frac{1}{2}}}<\infty .
$$

Moreover, Defant, Frerick, Ortega Cerdà, Ounaïes and Seip showed in [10 that the supremum of the set of all such real numbers numbers $C$ equals $\frac{1}{\sqrt{2}}$. This adds a level of precision that enables us to extract much more precise information about the coefficients of a Dirichlet series than what is obtained from the classical solution of the Bohr-Bohnenblust-Hille Theorem. Closely related to this, Maurizi and Queffélec observed in [25, Theorem 2.4] that the maximal

The first and third authors were supported by MICINN Project MTM2011-22417. 
width $S$ of Bohr's strip equals the infimum of all $\sigma \geq 0$ for which there exists a constant $C>0$ such that for all $N$ and all $a_{1}, \ldots, a_{N} \in \mathbb{C}$ we have

$$
\sum_{n=1}^{N}\left|a_{n}\right| \leq C N^{\sigma} \sup _{t \in \mathbb{R}}\left|\sum_{n=1}^{N} a_{n} n^{-i t}\right| .
$$

This motivates the following definition. Given a natural number $N$, let $Q_{N}$ be the best constant $D \geq 1$ such that for each choice of $a_{1}, \ldots, a_{N}$ in $\mathbb{C}$

$$
\sum_{n=1}^{N}\left|a_{n}\right| \leq D \sup _{t \in \mathbb{R}}\left|\sum_{n=1}^{N} a_{n} n^{-i t}\right| .
$$

The following result gives the asymptotically optimal upper and lower bound for $Q_{N}$, and it marks the endpoint of a long development started by Queffélec [27] in the mid nineties, continued by Konyagin and Queffélec [20, Theorem 4.3] in 2002 and by de la Bretèche [9. Théorèm 1.1] in 2008. The final result was proved in [10, Theorem 3]:

$$
Q_{N}=\frac{\sqrt{N}}{e^{\left(\frac{1}{\sqrt{2}}+o(1)\right) \sqrt{\log N \log \log N}}} .
$$

A key ingredient of the solution given by Bohnenblust and Hille to Bohr's problem was to consider $M$-homogeneous Dirichlet series $\sum_{n} a_{n} n^{-s}$, series for which $a_{n}=0$ for all indices $n$ which do not have exactly $M$ prime divisors according to their multiplicity. More precisely, if $n=p_{1}^{\alpha_{1}(n)} \ldots p_{r}^{\alpha_{r}(n)}$ is the prime factorization of $n$ and we write $\Omega(n):=\alpha_{1}(n)+\ldots+\alpha_{r}(n)$, then an $M$-homogeneous Dirichlet series is of the form $\sum_{\Omega(n)=M} a_{n} n^{-s}$. With this $S_{M}$ can be defined in the same way as $S$, just taking the supremum over all $M$-homogeneous Dirichlet series, and Bohnenblust and Hille showed that

$$
S_{M}=\frac{1}{2}-\frac{1}{2 M}
$$

The $M$-homogeneous analog of (1.1) was proved in [1, Theorem 1.4]: for every Dirichlet series in $\mathcal{H}_{M}^{\infty}$ (the Banach space of all $M$-homogeneous Dirichlet series in $\mathcal{H}^{\infty}$ ) we have

$$
\sum_{\substack{n=1 \\ \Omega(n)=M}}^{\infty}\left|a_{n}\right| \frac{(\log n)^{\frac{M-1}{2}}}{n^{\frac{M-1}{2 M}}}<\infty .
$$

From this and a careful analysis of the proof of [25. Theorem 3.1] it follows that (up to constants depending only on $M$ )

$$
Q_{N}^{M} \sim \frac{N^{\frac{M-1}{2 M}}}{(\log N)^{\frac{M-1}{2}}}
$$

clearly, $Q_{N}^{M}$ is here defined as above replacing arbitrary Dirichlet polynomials by $M$-homogeneous ones.

Let us turn to vector valued Dirichlet series $\sum_{n} a_{n} n^{-s}$, where the coefficients $a_{n}$ are in some Banach space $X$ (and $s$ still a complex variable). The study of the width of Bohr's strips for such objects was initiated in [11] and continued in [15]. Given a operator $v: X \rightarrow Y$ between two Banach spaces we define the number

$$
S(v):=\sup \left(\sigma_{a}(v D)-\sigma_{u}(D)\right),
$$


where the supremum is taken over all Dirichlet series $D$ in $X$. Here, given a Dirichlet series $D(s)=\sum a_{n} n^{-s}$ in $X, v D$ denotes the Dirichlet series $\sum v a_{n} n^{-s}$ in $Y$. The number $S_{M}(v)$ for $M \in \mathbb{N}$ is similarly defined considering only $M$-homogeneous instead of all Dirichlet series. If $v$ is the identity on $X$ we write $S(X)$ and $S_{M}(X)$. It turns out that for any finite dimensional $X$ we still have that $S(X)=\frac{1}{2}$ and $S_{M}(X)=\frac{M-1}{2 M}$. But if $X$ is infinite dimensional these two numbers coincide and depend only on the optimal cotype of $X$. More precisely, the main result from [11] shows that for any infinite dimensional Banach space $X$ (see Section 2 for the defintinion of $\cot (X))$

$$
S(X)=S_{M}(X)=1-\frac{1}{\cot (X)} .
$$

For the scale of $\ell_{p}$-spaces this gives

$$
S\left(\ell_{p}\right)=S_{M}\left(\ell_{p}\right)= \begin{cases}\frac{1}{2} & \text { if } 1 \leq p \leq 2 \\ 1-\frac{1}{p} & \text { if } 1 \leq p \leq \infty .\end{cases}
$$

This means that in infinite dimensional Banach spaces Bohr's strips do not distinguish between arbitrary and homogeneous Dirichlet series. In 15 this phenomenon was analysed for operators on the $\ell_{p}$-spaces. We have in [12, Corollary 5.7] and [15, Theorem 1.1] that for $1 \leq p<q \leq \infty$

$$
\begin{gathered}
S\left(\mathrm{id}: \ell_{p} \hookrightarrow \ell_{q}\right)= \begin{cases}\frac{1}{2} & \text { if } p \leq 2 \\
1-\frac{1}{p} & \text { if } p \geq 2 .\end{cases} \\
S_{M}\left(\mathrm{id}: \ell_{p} \hookrightarrow \ell_{q}\right)= \begin{cases}\frac{M-2\left(\frac{1}{p}-\max \left\{\frac{1}{q}, \frac{1}{2}\right\}\right)}{2 M} & \text { if } p \leq 2 \\
1-\frac{1}{p} & \text { if } p \geq 2 .\end{cases}
\end{gathered}
$$

Similarly, we know from [12, Corollary 5.9] and [14, Corollary 8.3] that for every operator $v: \ell_{1} \rightarrow \ell_{q}$ we have

$$
S(v)=\frac{1}{2} \text { and } S_{M}(v) \leq \begin{cases}\frac{M-2(1-1 / q)}{2 M} & \text { if } 1 \leq q \leq 2 \\ \frac{2 M(1-1 / q)-1}{2 M} & \text { if } 2 \leq q \leq \frac{2 M}{M-1} \\ \frac{1}{2} & \text { if } \frac{2 M}{M-1} \leq q \leq \infty\end{cases}
$$

Our main focus of this article is to give quantified versions of these vector valued results (1.7) - 1.10 similar to (1.4) and (1.6), and this will be done in terms of the following definition (motivated by 1.2 ) .

Definition 1.1. Given $N \in \mathbb{N}$ and an operator $v: X \rightarrow Y$ between Banach spaces, define $Q_{N}(v)$ to be the best constant $D \geq 1$ such that for each choice of $a_{1}, \ldots, a_{N} \in X$ we have

$$
\sum_{n=1}^{N}\left\|v a_{n}\right\|_{Y} \leq D \sup _{t \in \mathbb{R}}\left\|\sum_{n=1}^{N} a_{n} n^{-i t}\right\|_{X} .
$$

The $M$-homogeneous counterpart $Q_{N}^{M}(v)$ is defined by taking $a_{1}, \ldots, a_{N} \in X$ such that $a_{n}=0$ whenever $\Omega(n) \neq M$. If $v$ is the identity on $X$, the notation we use is $Q_{N}(X)$ and $Q_{N}^{M}(X)$.

Note first that the width $S(v)$ and $S_{M}(v)$ can be rephrased in terms of these numbers. A careful analysis of the proof of [25, Theorem 2.4] gives statement (1) in the following proposition. 


\section{Proposition 1.2.}

(1) $S(v)=\inf \left\{\sigma \geq 0 \mid \exists C_{\sigma} \forall N: Q_{N}(v) \leq C_{\sigma} N^{\sigma}\right\}$

(2) $S(v)=\limsup _{N \rightarrow \infty} \frac{\log Q_{N}(v)}{\log N}$

The corresponding results for $S_{M}(v)$ also hold.

Proof. It remains to prove (2); take an $s>\lim \sup \frac{\log Q_{N}(v)}{\log N}$. Clearly, there is an $N_{0}$ such that $\log Q_{N}(v) \leq \log N^{s}$ for every $N \geq N_{0}$. So there is a constant $C$ such that $Q_{N}(v) \leq C N^{s}$ for every $N$, which implies that $s \in\left\{\sigma \geq 0 \mid \exists C_{\sigma} \forall N: Q_{N}(v) \leq C_{\sigma} N^{\sigma}\right\}$. Hence

$$
\limsup _{N \rightarrow \infty} \frac{\log Q_{N}(v)}{\log N} \geq \inf \left\{\sigma \geq 0 \mid \exists C_{\sigma} \forall N: Q_{N}(v) \leq C_{\sigma} N^{\sigma}\right\}=S(v) .
$$

On the other hand, we know from (1) that for every $\sigma>S(v)$ there is a constant $C_{\sigma}$ such that $Q_{N}(v) \leq C_{\sigma} N^{\sigma}$ for every $N$, which implies that

$$
\limsup _{N \rightarrow \infty} \frac{\log Q_{N}(v)}{\log N} \leq \limsup _{N \rightarrow \infty}\left(\frac{\log C_{\sigma}}{\log N}+\frac{\sigma \log N}{\log N}\right)=\sigma
$$

which gives the conclusion.

We have the following general upper and lower estimates.

\section{Proposition 1.3.}

$$
\frac{\sqrt{N}}{e^{\left(\frac{1}{\sqrt{2}}+o(1)\right) \sqrt{\log N \log \log N}}} \leq Q_{N}(v) \leq\|v\| N
$$

Proof. The lower estimate is an immediate consequence of (1.4). The upper estimate follows from Carlson's equality (see [8] or [18, Lemma 3.2]): for every $a_{1}, \ldots, a_{N} \in \mathbb{C}$ we have

$$
\sum_{n=1}^{N}\left|a_{n}\right|^{2}=\lim _{T \rightarrow \infty} \frac{1}{2 T} \int_{-T}^{T}\left|\sum_{n=1}^{N} a_{n} n^{-i t}\right|^{2} d t .
$$

With this we get

$$
\begin{aligned}
& \sum_{n=1}^{N}\left\|v a_{n}\right\|_{Y} \leq N\|v\| \max _{1 \leq n \leq N}\left\|a_{n}\right\|_{X}=N\|v\| \max _{1 \leq n \leq N} \sup _{x^{\prime} \in B_{X^{\prime}}}\left|x^{\prime}\left(a_{n}\right)\right| \\
& \leq N\|v\| \sup _{x^{\prime} \in B_{X^{\prime}}}\left(\sum_{n=1}^{N}\left|x^{\prime}\left(a_{n}\right)\right|^{2}\right)^{\frac{1}{2}}=N\|v\| \sup _{x^{\prime} \in B_{X^{\prime}}}\left(\lim _{T \rightarrow \infty} \frac{1}{2 T} \int_{-T}^{T}\left|\sum_{n=1}^{N} x^{\prime}\left(a_{n}\right) n^{-i t}\right|^{2} d t\right)^{\frac{1}{2}} \\
& \leq N\|v\| \sup _{x^{\prime} \in B_{X^{\prime}}} \sup _{t \in \mathbb{R}}\left|x^{\prime}\left(\sum_{n=1}^{N} a_{n} n^{-i t}\right)\right|=N\|v\| \sup _{t \in \mathbb{R}}\left\|\sum_{n=1}^{N} a_{n} n^{-i t}\right\|_{X} .
\end{aligned}
$$

It turns out that for more concrete operators the general estimate in Proposition 1.3 can be improved considerably (see Theorems 3.1, 6.1 and 6.2). 


\section{Preliminaries}

By $a_{N} \ll b_{N}$ we mean that there is a constant $c>0$ such that $a_{N} \leq c b_{N}$ for every $N \in \mathbb{N}$, and whenever $a_{N} \ll b_{N}$ and $b_{N} \ll a_{N}$, then we write $a_{N} \sim b_{N}$. Moreover, $a_{N} \ll b_{N}$ means that $a_{N} \leq c b_{N}$ for some constant $c$ and every $N$; if $a_{N} \ll b_{N}$ and $b_{N} \ll a_{N}$ then we write $a_{N} \sim b_{N}$. We already indicated that if $n=p_{1}^{\alpha_{1}(n)} \cdots p_{r}^{\alpha_{r}(n)}$ is the prime factorization of $n \in \mathbb{N}$, then $\Omega(n):=\alpha_{1}(n)+\ldots+\alpha_{r}(n)$. As usual, $\pi(n)$ denotes the number of primes $p \leq$. We use standard notation and notions from Banach space theory, as presented e.g. in [23, 24]. All Banach spaces $X$ are assumed to be complex, their duals are denoted by $X^{\prime}$ and their open unit balls by $B_{X}$. The conjugate exponent $p^{*}$ for $1 \leq p \leq \infty$ is as usual defined by $1=\frac{1}{p}+\frac{1}{p^{*}}$. A Banach lattice $X$ is said to be $q$-concave, $1 \leq q<\infty$, if there is a constant $C>0$ such that for every choice of finitely many $x_{1}, \ldots, x_{N} \in X$ we have

$$
\left(\sum_{n=1}^{N}\left\|x_{n}\right\|^{q}\right)^{\frac{1}{q}} \leq C\left\|\left(\sum_{n=1}^{N}\left|x_{n}\right|^{q}\right)^{\frac{1}{q}}\right\| ;
$$

The best such $C$ is as usual denoted by $M_{p}(X)$.

A function $P: X \rightarrow Y$ between two Banach spaces is said to be a (continuous) $M$-homogeneous polynomial if there is a (continuous) $M$-linear mapping $A: X \times \ldots \times X \rightarrow Y$ such that $P(x)=$ $A(x, \ldots, x)$ for all $x \in X$. We denote by $\mathcal{P}\left({ }^{M} X, Y\right)$ the vector space of all $M$-homogeneous continuous polynomials $P: X \rightarrow Y$ which together with the norm $\|P\|=\sup _{x \in B_{X}}\|P(x)\|_{Y}$ forms a Banach space.

An operator $v: X \rightarrow Y$ is $(p, q)$-summing, $1 \leq p, q<\infty$, if there is a constant $C>0$ such that for each choice of finitely many $x_{1}, \ldots, x_{N} \in X$ we have

$$
\left(\sum_{n=1}^{N}\left\|v x_{n}\right\|^{p}\right)^{1 / p} \leq C \sup _{x^{\prime} \in B_{X^{\prime}}}\left(\sum_{n=1}^{N}\left|x^{\prime}\left(x_{n}\right)\right|^{q}\right)^{\frac{1}{q}} ;
$$

for the best such $C$ we write $\pi_{p, q}(v)$.

A Banach space $X$ is said to have cotype $q$, where $2 \leq q \leq \infty$, if there exists a constant $C>0$ such that for every choice of finitely many vectors $x_{1}, \ldots, x_{N} \in X$ we have

$$
\left(\sum_{n=1}^{N}\left\|x_{n}\right\|^{q}\right)^{1 / q} \leq C\left(\int_{0}^{1}\left\|\sum_{n=1}^{N} r_{n}(t) x_{n}\right\|^{2} d t\right)^{1 / 2},
$$

where $r_{n}$ stands for the $n$th Rademacher function on $[0,1]$; the best such $C$ is denoted by $C_{q}(X)$. We write $\cot (X):=\inf \{2 \leq q \leq \infty \mid X$ has cotype $q\}$. Note that every Banach space has cotype $\infty$. In particular, it is well known that

$$
\cot \left(\ell_{p}\right)= \begin{cases}2 & p \leq 2 \\ p & p>2\end{cases}
$$

We finish this section by defining for each $N$ and $M$ the index sets

$$
\begin{gathered}
\mathcal{M}(M, N)=\left\{\left(i_{1}, \ldots, i_{M}\right) \mid 1 \leq i_{k} \leq N \text { for each } k\right\}=\{1, \ldots, N\}^{M} \\
\mathcal{J}(M, N)=\left\{i \in \mathcal{M}(M, N) \mid i_{1} \leq \ldots \leq i_{M}\right\} \\
\Lambda(M, N)=\left\{\alpha \in \mathbb{N}_{0}^{N}|| \alpha \mid=M\right\}
\end{gathered}
$$

There is a one-to-one correspondence between $\Lambda(M, N)$ and $\mathcal{J}(M, N)$ : for each $\alpha \in \Lambda(M, N)$ the associated index $\mathbf{j}_{\alpha} \in \mathcal{J}(M, N)$ is given by $\mathbf{j}_{\alpha}=\left(1,{ }_{1}{ }_{1}, 1,2,{ }_{2} .{ }_{2}, 2, \ldots, N,{ }_{N} ., N\right)$ and 
on the other hand for $\mathbf{j} \in \mathcal{J}(M, N)$ the associated multi-index $\mathbf{j}_{\alpha} \in \Lambda(M, N)$ is given by $\mathbf{j}_{\alpha_{r}}=\left|\left\{k \mid j_{k}=r\right\}\right|$. We define the following equivalence relation in $\mathcal{M}(M, N): \mathbf{i} \sim \mathbf{j}$ if there is a permutation $\sigma$ such that $i_{\sigma(k)}=j_{k}$ for all $1 \leq k \leq M$, then $\mathcal{M}(M, N)=\dot{\bigcup}_{\mathbf{j} \in \mathcal{J}(M, N)}[\mathbf{j}]$. Note that $\operatorname{card}\left[\mathbf{j}_{\alpha}\right]=\frac{M !}{\alpha !}$ for every $\alpha \in \Lambda(M, N)$.

Our proofs involve techniques of complex analysis, number theory, local Banach space theory and probability theory. One crucial ingredient is the following ingenious idea of Harald Bohr relating the theory of Dirichlet series with infinite dimensional holomorphy. This fact was rediscovered and systematised by Hedenmalm, Linqvist and Seip [18]: the mapping

$$
\begin{array}{ccc}
\mathfrak{B}: & H_{\infty}\left(B_{c_{0}}\right) \longrightarrow \mathcal{H}^{\infty} \\
& \sum_{\alpha} c_{\alpha} z^{\alpha} \stackrel{c_{\alpha}=a_{p^{\alpha}}}{\longrightarrow} \sum_{n} a_{n} n^{-s}
\end{array}
$$

defines an isometric isomorphism. Here $H_{\infty}\left(B_{c_{0}}\right)$ denotes the Banach space of all bounded, holomorphic (i.e. complex Fréchet differentiable) functions on the open unit ball $B_{c_{0}}$ of $c_{0}$. In the vector valued case, using the Hahn-Banach theorem we have that $H_{\infty}\left(B_{c_{0}}, X\right)$ and $\mathcal{H}^{\infty}(X)$ (both spaces defined in the obvious way) are isometrically isomorphic; in particular for every finite choice of $a_{1}, \ldots, a_{N} \in X$ we have

$$
\sup _{t \in \mathbb{R}}\left\|\sum_{n=1}^{N} a_{n} n^{i t}\right\|_{X}=\sup _{z \in \mathbb{D}^{N}}\left\|\sum_{n=1}^{N} a_{n} z_{1}^{\alpha_{1}(n)} \cdots z_{r}^{\alpha_{r}(n)}\right\|_{X} .
$$

Our aim is to give estimates of $Q_{N}(v)$ and $Q_{N}^{M}(v)$ for certain concrete operators. We will obtain general upper and lower estimates involving $(r, 1)$-summing operators. Then the estimates for concrete operators between $\ell_{p}$ spaces (Theorems 6.1 and 6.2 will follow from these general estimates (Theorems 4.1 and 4.2 , together with the following two well-known Grothendieck-type inequalities:

- Bennett-Carl inequalities [3,7]: for $1 \leq p \leq q \leq \infty$ the embedding id : $\ell_{p} \hookrightarrow \ell_{q}$, is $(r, 1)$-summing with $\frac{1}{r}=\frac{1}{2}+\frac{1}{p}-\max \left\{\frac{1}{q}, \frac{1}{2}\right\}$. A forerunner of this result is Littlewood's $\frac{4}{3}$-inequality stating that id $: \ell_{1} \hookrightarrow \ell_{\frac{4}{3}}$ is $\left(\frac{4}{3}, 1\right)$-summing.

- Kwapień's theorem [21]: every linear operator $v: \ell_{1} \rightarrow \ell_{q}$ is $(r, 1)$-summing for $\frac{1}{r}=1-\left|\frac{1}{q}-\frac{1}{2}\right|$. The case $q=2$ is Grothendieck's theorem.

In both results the corresponding $r$ is known to be optimal.

\section{Estimates FOR IDENTITY OPERATORS}

In view of Proposition 1.2, our first result is a quantified version of (1.7).

Theorem 3.1. Let $X$ be a Banach space. Then with constants depending only on $X$ we have

$$
\begin{gathered}
Q_{N}(X)=\frac{\sqrt{N}}{e^{\left(\frac{1}{\sqrt{2}}+o(1)\right) \sqrt{\log N \log \log N}}} \\
Q_{N}^{M}(X) \sim \frac{N^{\frac{M-1}{2 M}}}{(\log N)^{\frac{M-1}{2}}}
\end{gathered}
$$


provided $X$ is finite dimensional. Whereas if $X$ is infinite dimensional, then (with constants depending on $X$ and and $\varepsilon$ )

$$
\begin{gathered}
N^{1-\frac{1}{\cot (X)}} \ll Q_{N}(X) \ll N^{1-\frac{1}{\cot (X)+\varepsilon}} \\
\left(\frac{N}{\log N}\right)^{1-\frac{1}{\cot (X)}} \ll Q_{N}^{M}(X) \ll N^{1-\frac{1}{\cot (X)+\varepsilon}} .
\end{gathered}
$$

Proof. We suppose first that $X$ is finite dimensional; then the identity id $X$ is absolutely $(1,1)$-summing. Hence for every choice of $a_{1}, \ldots, a_{N} \in X$ we have

$$
\begin{aligned}
\sum_{n=1}^{N}\left\|a_{n}\right\| \leq \pi_{1,1}\left(\operatorname{id}_{X}\right) \sup _{x^{\prime} \in B_{X^{\prime}}} \sum_{n=1}^{N}\left|x^{\prime}\left(a_{n}\right)\right| \leq \pi_{1,1}\left(\operatorname{id}_{X}\right) \sup _{x^{\prime} \in B_{X^{\prime}}} Q_{N} \sup _{t \in \mathbb{R}}\left|\sum_{n=1}^{N} x^{\prime}\left(a_{n}\right) n^{-i t}\right| \\
=\pi_{1,1}\left(\operatorname{id}_{X}\right) Q_{N} \sup _{t \in \mathbb{R}} \sup _{x^{\prime} \in B_{X^{\prime}}}\left|x^{\prime}\left(\sum_{n=1}^{N} a_{n} n^{-i t}\right)\right|=\pi_{1,1}\left(\operatorname{id}_{X}\right) Q_{N} \sup _{t \in \mathbb{R}}\left\|\sum_{n=1}^{N} a_{n} n^{-i t}\right\| .
\end{aligned}
$$

This gives the upper estimate in (3.1). The proof for $(3.2)$ follows the same lines. The lower estimates both in (3.1) and (3.2) follow from the finite dimensional case (1.4). Let us assume now that $X$ is infinite dimensional and let us give the upper estimate in (3.3). We know from (1.7) and Proposition 1.2 that

$$
1-\frac{1}{\cot (X)}=S(X)=\inf \left\{\sigma \geq 0 \mid \exists C_{\sigma} \forall N: Q_{N}(X) \leq C_{\sigma} N^{\sigma}\right\} .
$$

Hence for every $q>\cot (X)$ there is a constant $C_{q}$ such that for all $N$

$$
Q_{N}(X) \leq C_{q} N^{1-\frac{1}{q}}
$$

This completes the proof of the upper bound; the $M$-homogeneous case follows in the same way.

Finally, given $X$ let us take $r<\cot (X)$. By [16, Theorem 14.5] for every $0<\varepsilon<1$ and for every $N$ there are $x_{1}, \ldots, x_{N} \in X$ so that for every $z \in \mathbb{C}^{N}$

$$
\frac{1}{1+\varepsilon}\|z\|_{\infty} \leq\left\|\sum_{n=1}^{N} z_{n} x_{n}\right\|_{X} \leq\|z\|_{r} .
$$

In particular, taking $z=e_{n}$ we have $\frac{1}{1+\varepsilon} \leq\left\|x_{n}\right\|$. Then we have

$$
\begin{aligned}
& \frac{N}{1+\varepsilon} \leq \sum_{n=1}^{N}\left\|x_{n}\right\| \leq Q_{N}(X) \sup _{t \in \mathbb{R}}\left\|\sum_{n=1}^{N} x_{n} n^{i t}\right\| \\
& \leq Q_{N}(X) \sup _{z \in \mathbb{D}^{N}}\left\|\sum_{n=1}^{N} x_{n} z_{n}\right\| \leq Q_{N}(X) \sup _{z \in \mathbb{D}^{N}}\|z\|_{r}=Q_{N}(X) N^{\frac{1}{r}} .
\end{aligned}
$$

This implies $\frac{N^{1-\frac{1}{r}}}{1+\varepsilon} \leq Q_{N}(X)$ for every $0<\varepsilon<1$, hence $N^{1-\frac{1}{r}} \leq Q_{N}(X)$. Since this holds for every $r<\cot (X)$ we get the lower estimate in (3.3). For (3.4) let us define $a_{p_{n}}=x_{n}$ for 
$n=1, \ldots, N$ (and 0 otherwise) and consider the Dirichlet series $\sum a_{k} k^{-s}$. We have by 2.2 )

$$
\begin{aligned}
& \frac{N}{1+\varepsilon} \leq \sum_{n=1}^{N}\left\|a_{p_{n}}\right\| \leq Q_{p_{N}}^{1}(X) \sup _{t \in \mathbb{R}}\left\|\sum_{n=1}^{N} a_{p_{n}} p_{n}^{i t}\right\| \\
& \quad=Q_{p_{N}}^{1}(X) \sup _{z \in \mathbb{D}^{N}}\left\|\sum_{n=1}^{N} x_{n} z_{n}\right\| \leq Q_{p_{N}}^{1}(X) \sup _{z \in \mathbb{D}^{N}}\|z\|_{q}=Q_{p_{N}}^{1}(X) N^{\frac{1}{q}} .
\end{aligned}
$$

Proceeding as before and by the Prime Number Theorem we have

$$
\left(\frac{N}{\log N}\right)^{1-\frac{1}{q}} \ll Q_{N}^{1}(X) \leq Q_{N}^{M}(X)
$$

as desired.

Remark 1. For $\ell_{p}$-spaces with $2 \leq p \leq \infty$ we can improve the upper estimate given in (3.3) getting $Q_{N}\left(\ell_{p}\right) \leq N^{1-\frac{1}{p}}$. Indeed, the case $p=\infty$ is shown in Proposition 1.3 . For $2 \leq p<\infty$ we have

$$
\begin{aligned}
\left(\sum_{n=1}^{N}\left\|a_{n}\right\|_{p}\right)^{\frac{1}{p}}=\left(\sum_{l=1}^{\infty} \sum_{n=1}^{N}\left|a_{n}(l)\right|^{p}\right)^{\frac{1}{p}} & \leq\left(\sum_{l=1}^{\infty}\left(\sum_{n=1}^{N}\left|a_{n}(l)\right|^{2}\right)^{\frac{p}{2}}\right)^{\frac{1}{p}} \\
& =\left(\sum_{l=1}^{\infty} \lim _{T \rightarrow \infty}\left(\frac{1}{2 T} \int_{-T}^{T}\left|\sum_{n=1}^{N} a_{n}(l) n^{i t}\right|^{2} d t\right)^{\frac{p}{2}}\right)^{\frac{1}{p}},
\end{aligned}
$$

Now, for each fixed $L$ we have, using Minkowski's inequality

$$
\begin{aligned}
& \left(\sum_{l=1}^{L} \lim _{T \rightarrow \infty}\left(\frac{1}{2 T} \int_{-T}^{T}\left|\sum_{n=1}^{N} a_{n}(l) n^{i t}\right|^{2} d t\right)^{\frac{p}{2}}\right)^{\frac{1}{p}}=\lim _{T \rightarrow \infty}\left(\sum_{l=1}^{L}\left(\frac{1}{2 T} \int_{-T}^{T}\left|\sum_{n=1}^{N} a_{n}(l) n^{i t}\right|^{2} d t\right)^{\frac{p}{2}}\right)^{\frac{1}{p}} \\
& \quad \leq \lim _{T \rightarrow \infty}\left(\frac{1}{2 T} \int_{-T}^{T}\left(\sum_{l=1}^{L}\left|\sum_{n=1}^{N} a_{n}(l) n^{i t}\right|^{p}\right)^{\frac{2}{p}} d t\right)^{\frac{1}{2}} \leq\left(\lim _{T \rightarrow \infty} \frac{1}{2 T} \int_{-T}^{T}\left\|\sum_{n=1}^{N} a_{n} n^{i t}\right\|_{p}^{2} d t\right)^{\frac{1}{2}} \\
& \quad \leq \sup _{t \in \mathbb{R}}\left\|\sum_{n=1}^{N} a_{n} n^{i t}\right\|_{p} .
\end{aligned}
$$

Since this holds for every $L$ we have, by Hölder's inequality,

$$
\sum_{n=1}^{N}\left\|a_{n}\right\|_{p} \leq N^{1-\frac{1}{p}}\left(\sum_{n=1}^{N}\left\|a_{n}\right\|^{p}\right)^{\frac{1}{p}} \leq N^{1-\frac{1}{p}} \sup _{t \in \mathbb{R}}\left\|\sum_{n=1}^{N} a_{n} n^{i t}\right\|_{p}
$$

\section{UPPER ESTIMATES}

For our first upper estimate we follow the proof of Konyagin and Queffélec [20] as presented by de la Bretèche in [9] for the scalar case together with its improvement of Defant, Frerick, Ortega-Cerdà, Ounaïes, and Seip in [10]. The crucial point there is the so called hypercontractivity of the polynomial Bohnenblust-Hille inequality. We will use a vector valued variant of this inequality [13, Theorem 5.3].

Note that, although the setting here is quite general, when $v=\operatorname{id}_{\mathbb{C}}$ then $q=2$ and $r=1$, we 
recover the scalar result (1.4).

Theorem 4.1. Let $Y$ be a q-concave Banach lattice with $2 \leq q<\infty$ and $v: X \rightarrow Y$ an $(r, 1)$-summing operator with $1 \leq r<q$. Then

$$
Q_{N}(v) \leq \frac{N^{1-\frac{1}{q}}}{e^{\left(2 \frac{q-1}{q} \sqrt{\frac{1}{r}-\frac{1}{q}}+o(1)\right) \sqrt{\log N \log \log N}}}
$$

Proof. For any natural $n$ let $P^{+}(n)$ be the largest prime factor of $n$ and $P^{-}(n)$ the smallest prime factor of $n$, with the convention $P^{+}(1)=P^{-}(1)=1$. For $M, N \in \mathbb{N}$ and $y \leq N$ define the following sets

$$
\begin{aligned}
S(N, y) & :=\left\{n \leq N \mid P^{+}(n) \leq y\right\} \\
T(N, y) & :=\left\{n \leq N \mid P^{-}(n)>y\right\} \\
T_{M}(N, y) & :=\{n \in T(N, y) \mid \Omega(n)=M\} .
\end{aligned}
$$

By [9, Lemme 2.3] there is an absolute constant $D>0$ such that for any $M, N, y$

$$
\left|T_{M}(N, y)\right| \ll \frac{N}{y^{M}}(\log N)^{y-1} e^{D y} .
$$

Note that for any $y \leq N$ each $n \in\{1, \ldots, N\}$ can be uniquely decomposed as

$$
n=k l, \quad \text { where } k \in S(N, y) \text { and } l \in T\left(\frac{N}{k}, y\right) \text {. }
$$

Given a Dirichlet polynomial $D=\sum_{n=1}^{N} a_{n} n^{-s}$ in $X$, let $P=\mathfrak{B}^{-1}(D)$ be the associated polynomial $P(z)=\sum_{n=1}^{N} a_{n} z_{1}^{\alpha_{1}(n)} \cdots z_{d}^{\alpha_{d}(n)}$ with $d=\pi(N)$ (the cardinality of all primes $\leq N)$. With the decomposition from 4.2 we have

$$
P(z)=\sum_{n=1}^{N} a_{n} z_{1}^{\alpha_{1}(n)} \cdots z_{d}^{\alpha_{d}(n)}=\sum_{k \in S(N, y)} z_{1}^{\alpha_{1}(k)} \cdots z_{s}^{\alpha_{s}(k)} \sum_{l \in T\left(\frac{N}{k}, y\right)} a_{k l} z_{s+1}^{\alpha_{s+1}(l)} \cdots z_{d}^{\alpha_{d}(l)},
$$

where $s=\pi(y)$. For $k \in S(N, y)$ define

$$
P_{k}\left(z_{s+1}, \ldots, z_{d}\right)=\sum_{l \in T\left(\frac{N}{k}, y\right)} a_{k l} z_{s+1}^{\alpha_{s+1}(l)} \cdots z_{d}^{\alpha_{d}(l)},
$$

and denote by $P_{k}^{(M)}$ the $M$-homogeneous part of $P_{k}$. Following [20], an easy calculation shows that

$$
P_{k}(z)=\frac{1}{(2 \pi)^{s}} \int_{0}^{2 \pi} \ldots \int_{0}^{2 \pi} P\left(e^{i t_{1}}, \ldots, e^{i t_{s}}, z\right) e^{-i\left(\alpha_{1}(k) t_{1}+\ldots+\alpha_{s}(k) t_{s}\right)} d t_{1} \ldots d t_{s} .
$$

This and Cauchy's inequalities give

$$
\left\|P_{k}^{(M)}\right\| \leq\left\|P_{k}\right\| \leq\|P\| .
$$

We have

$$
\sum_{n=1}^{N}\left\|v a_{n}\right\|_{Y}=\sum_{k \in S(N, y)} \sum_{M \geq 1} \sum_{l \in T_{M}\left(\frac{N}{k}, y\right)}\left\|v a_{k l}\right\| \leq|S(N, k)| \sup _{k \in S(N, y)} \sum_{M \geq 1} \sum_{l \in T_{M}\left(\frac{N}{k}, y\right)}\left\|v a_{k l}\right\|_{Y} .
$$


Using Hölder's inequality with $\rho_{M}=\frac{q r M}{q+(M-1) r}$ and $\rho_{M}^{*}=\frac{q M}{(q-1) M-\frac{q}{r}+1}$ we get

$$
\sum_{l \in T_{M}\left(\frac{N}{k}, y\right)}\left\|v a_{k l}\right\|_{Y} \leq\left(\sum_{l \in T_{M}\left(\frac{N}{k}, y\right)}\left\|v a_{k l}\right\|_{Y}^{\rho_{M}}\right)^{\frac{1}{\rho_{M}}}\left|T_{M}(N, y)\right|^{\frac{1}{\rho_{M^{*}}}}
$$

By [13, Theorem 5.3] there is an absolute constant $C>0$ such that

$$
\left(\sum_{l \in T_{M}\left(\frac{N}{k}, y\right)}\left\|v a_{k l}\right\|_{Y}^{\rho_{M}}\right)^{\frac{1}{\rho_{M}}} \leq C^{M}\left\|P_{k}^{(M)}\right\|
$$

Then 4.3, 2.2 and 4.1 give

$$
\begin{aligned}
\sum_{l \in T_{M}\left(\frac{N}{k}, y\right)}\left\|v a_{k l}\right\|_{Y} & \leq C^{M}\|P\|\left|T_{M}(N, y)\right|^{\frac{1}{\rho_{M}}} \\
& \ll C^{M} \sup _{t \in \mathbb{R}}\left\|\sum_{n=1}^{N} a_{n} n^{i t}\right\|\left(\frac{N}{y^{M}}(\log N)^{y-1} e^{D y}\right)^{\frac{q-1}{q}-\frac{1}{M}\left(\frac{1}{r}-\frac{1}{q}\right)} \\
& \leq N^{\frac{q-1}{q}} e^{h_{N, y}^{q, r}(M)} \sup _{t \in \mathbb{R}}\left\|\sum_{n=1}^{N} a_{n} n^{i t}\right\|,
\end{aligned}
$$

where

$$
\begin{aligned}
h_{N, y}^{q, r}(x):=x \log C-\left(\frac{1}{r}-\frac{1}{q}\right) \frac{1}{x} \log N-\frac{q-1}{q} x \log y \\
\quad+\left(\frac{1}{r}-\frac{1}{q}\right) \log y+\frac{q-1}{q}(y-1) \log \log N+\frac{q-1}{q} D y .
\end{aligned}
$$

This holds for every $y \leq N$; we take $y=\frac{\sqrt{\log N}}{\log \log N}$ and by differentiating and maximizing we have that, for $N$ big enough

$$
h_{N, y}^{r, q}(M) \leq\left(-\sqrt{2 \frac{q-1}{q}\left(\frac{1}{r}-\frac{1}{q}\right)}+o(1)\right) \sqrt{\log N \log \log N}
$$

for every $M$. Then

$$
\sum_{l \in T_{M}\left(\frac{N}{k}, y\right)}\left\|v a_{k l}\right\|_{Y} \ll N^{\frac{q-1}{q}} \sup _{t \in \mathbb{R}}\left\|\sum_{n=1}^{N} a_{n} n^{i t}\right\| e^{\left(-\sqrt{2 \frac{q-1}{q}\left(\frac{1}{r}-\frac{1}{q}\right)}+o(1)\right) \sqrt{\log N \log \log N}} .
$$

On the other hand as in $|9|$ we use that $\left|S\left(N, \frac{\sqrt{\log N}}{\log \log N}\right)\right| \leq e^{\mu \frac{\sqrt{\log N}}{\log \log N}}$ for some $\mu>0$. Since $N$ has at most $\frac{\log N}{\log 2}$ prime factors, we have that $T_{M}\left(\frac{N}{k}, y\right)=\emptyset$ for each $M>\frac{\log N}{\log 2}$. Summarizing 
we finally have

$$
\begin{gathered}
\sum_{n=1}^{N}\left\|v a_{n}\right\|_{Y} \ll\left|S\left(N, \frac{\sqrt{\log N}}{\log \log N}\right)\right| \sup _{k \in S(N, y)} \sum_{M \geq 1} \sum_{l \in T_{M}\left(\frac{N}{k}, y\right)}\left\|v a_{k l}\right\|_{Y} \\
\leq N^{\frac{q-1}{q}} \sup _{t \in \mathbb{R}}\left\|\sum_{n=1}^{N} a_{n} n^{i t}\right\| e^{\mu \frac{\sqrt{\log N} \log N}{\log } \frac{\log N}{\log 2}+\left(-\sqrt{2 \frac{q-1}{q}\left(\frac{1}{r}-\frac{1}{q}\right)}+o(1)\right) \sqrt{\log N \log \log N}} \\
\left.=N^{\frac{q-1}{q}} \sup _{t \in \mathbb{R}}\left\|\sum_{n=1}^{N} a_{n} n^{i t}\right\| e^{\left(-\sqrt{2 \frac{q-1}{q}\left(\frac{1}{r}-\frac{1}{q}\right)}+o(1)\right.}\right) \sqrt{\log N \log \log N}
\end{gathered}
$$

We now present a general upper estimate for the $M$-homogeneous case.

Theorem 4.2. Let $Y$ be a q-concave Banach lattice with $2 \leq q<\infty$ and $v: X \rightarrow Y$ an $(r, 1)$-summing operator with $1 \leq r<q$. Then for every $0<\lambda<\frac{q-1}{q}(M-1)$ there is $D>0$ such that for every $N$ and every $M$-homogeneous Dirichlet series $\sum a_{n} n^{-s}$

$$
\sum_{n=1}^{N}\left\|v a_{n}\right\|_{Y} \frac{(\log n)^{\lambda}}{n^{\frac{(q-1) M-q\left(\frac{1}{r}-\frac{1}{q}\right)}{q M}}} \leq D \sup _{t \in \mathbb{R}}\left\|\sum_{n=1}^{N} a_{n} n^{-i t}\right\|_{X}
$$

For the proof we need the following Lemma.

Lemma 4.3. With the assumptions of Theorem 4.2, for every $N$ and every $M$-homogeneous polynomial $P \in \mathcal{P}\left({ }^{M} \ell_{\infty}^{N}, X\right), P(z)=\sum b_{j_{1} \ldots j_{M}} z_{j_{1}} \cdots z_{j_{M}}$ we have

$$
\left(\sum_{j_{M}=1}^{N}\left(\sum_{\substack{j_{1}, \ldots, j_{M-1}: \\ j_{1} \leq \ldots \leq j_{M}}}\left\|v b_{j_{1} \ldots j_{M}}\right\|_{Y}^{q}\right)^{\frac{r}{q}}\right)^{\frac{1}{r}} \leq C \sup _{z \in_{B} \ell_{\infty}^{N}}\left\|\sum_{\mathbf{j} \in \mathcal{J}(M, N)} b_{j_{1} \ldots j_{M}} z_{j_{1}} \cdots z_{j_{M}}\right\|,
$$

where $C=M M_{q}(Y) \sqrt{2}^{M-1} \pi_{r, 1}(v)\left(1+\frac{1}{M-1}\right)^{M-1}$.

Proof. Let $P \in \mathcal{P}\left({ }^{M} \ell_{\infty}^{N}, X\right)$ be an $M$-homogeneous polynomial, and $A$ its associated symmetric $M$-linear mapping such that $P(z)=A(z, \ldots, z)$. It is well know that the monomial coefficients $b_{j_{1} \ldots j_{M}}$ of $P$ and the coefficients $a_{i_{1} \ldots i_{M}}:=A\left(e_{i_{1}}, \ldots, e_{i_{M}}\right)$ defining $A$ are related in the following way

$$
b_{j_{1} \ldots j_{M}}=\operatorname{card}[\mathbf{j}] a_{j_{1} \ldots j_{M}} .
$$

On the other hand, for each $\mathbf{j}=\left(j_{1}, \ldots, j_{M-1}\right) \in \mathcal{J}(M-1, N)$ and $1 \leq i_{M} \leq N$ we write $\mathbf{j}, i_{M}=\left(j_{1}, \ldots, j_{M-1}, i_{M}\right)$ and have

$$
\frac{\operatorname{card}\left[\mathbf{j}, i_{M}\right]}{\operatorname{card}[\mathbf{j}]}=\frac{M !}{(M-1) !} \cdot \frac{\left|\left\{k \mid \mathbf{j}_{k}=1\right\}\right| !}{\left|\left\{k \mid\left(\mathbf{j}, i_{M}\right)_{k}=1\right\}\right| !} \cdots \frac{\left|\left\{k \mid \mathbf{j}_{k}=N\right\}\right| !}{\left|\left\{k \mid\left(\mathbf{j}, i_{M}\right)_{k}=N\right\}\right| !} \leq M .
$$


With this, using the fact that $Y$ is $q$-concave, we get

$$
\begin{aligned}
\left(\sum_{j_{M}=1}^{N}\left(\sum_{\substack{j_{1}, \ldots, j_{M-1}: \\
j_{1} \leq \ldots \leq j_{M}}}\left\|v b_{j_{1} \ldots j_{M}}\right\|_{Y}^{q}\right)^{\frac{r}{q}}\right)^{\frac{1}{r}}=\left(\sum_{i_{M}=1}^{N}\left(\sum_{\substack{j_{1}, \ldots, j_{M-1}: \\
j_{1} \leq \ldots \leq i_{M}}}\left\|\operatorname{card}\left[\mathbf{j}, i_{M}\right] v a_{\mathbf{j}, i_{M}}\right\|_{Y}^{q}\right)^{\frac{r}{q}}\right)^{\frac{1}{r}} \\
\leq\left(\sum_{i_{M}=1}^{N}\left(\sum_{\mathbf{j} \in \mathcal{J}(M-1, N)}\left\|\operatorname{card}\left[\mathbf{j}, i_{M}\right] v a_{\left.\mathbf{j}, i_{M}\right)}\right\|_{Y}^{q}\right)^{\frac{r}{q}}\right)^{\frac{1}{r}} \\
\leq M\left(\sum_{i_{M}=1}^{N}\left(\sum_{\mathbf{j} \in \mathcal{J}(M-1, N)}\left\|\operatorname{card}[\mathbf{j}] v a_{\left.\mathbf{j}, i_{M}\right)}\right\|_{Y}^{q}\right)^{\frac{r}{q}}\right)^{\frac{1}{r}} \\
\leq M M_{q}(Y)\left(\sum_{i_{M}=1}^{N}\left\|\left(\sum_{\mathbf{j} \in \mathcal{J}(M-1, N)}\left|\operatorname{card}[\mathbf{j}] v a_{\mathbf{j}, i_{M}}(l)\right|^{q}\right)^{\frac{1}{q}}\right\|_{Y}^{r}\right)^{\frac{1}{r}} \\
\leq M M_{q}(Y)\left(\sum_{i_{M}=1}^{N}\left\|\left(\sum_{\mathbf{j} \in \mathcal{J}(M-1, N)}\left|\operatorname{card}[\mathbf{j}] v a_{\mathbf{j}, i_{M}}(l)\right|^{2}\right)^{\frac{1}{2}}\right\|_{Y}^{r}\right)^{\frac{1}{r}}
\end{aligned}
$$

Let now $\mu^{N}$ denote the normalized Lebesgue measure on $\mathbb{T}^{N}$. By [2, Theorem 9] (see also [10, Lemma 2]) for every $P \in \mathcal{P}\left({ }^{M} \ell_{\infty}^{N}, \mathbb{C}\right), P(z)=\sum_{\alpha \in \Lambda(M, N)} c_{\alpha} z^{\alpha}$ we have

$$
\left(\sum_{\alpha \in \Lambda(M, N)}\left|c_{\alpha}\right|^{2}\right)^{\frac{1}{2}} \leq \sqrt{2}^{M} \int_{\mathbb{T}^{N}}\left|\sum_{\alpha \in \Lambda(M, N)} c_{\alpha} z^{\alpha}\right| d \mu^{N}(z) .
$$

Applying Krivine's calculus (as presented e.g. in [24, pp. 40-42]), this inequality also holds in Banach lattices. Hence by Minkowski's integral inequality,

$$
\begin{aligned}
\left(\sum_{i_{M}=1}^{N} \|\right. & \left.\left(\sum_{\mathbf{j} \in \mathcal{J}(M-1, N)}\left|\operatorname{card}[\mathbf{j}] v a_{\mathbf{j}, i_{M}}(l)\right|^{2}\right)^{\frac{1}{2}} \|_{Y}^{r}\right)^{\frac{1}{r}} \\
& \leq \sqrt{2}^{M-1}\left(\sum_{i_{M}=1}^{N}\left\|\int_{\mathbb{T}^{N}}\left|\sum_{\mathbf{j} \in \mathcal{J}(M-1, N)} \operatorname{card}[\mathbf{j}] v a_{\mathbf{j}, i_{M}}(l) z_{j_{1}} \cdots z_{j_{M-1}}\right| d \mu^{N}(z)\right\|_{Y}\right)^{\frac{1}{r}} \\
& \leq \sqrt{2}^{M-1} \int_{\mathbb{T}^{N}}\left(\sum_{i_{M}=1}^{N}\left\|\sum_{\mathbf{j} \in \mathcal{J}(M-1, N)} \operatorname{card}[\mathbf{j}] v a_{\mathbf{j}, i_{M}} z_{j_{1}} \cdots z_{j_{M-1}}\right\|_{Y}^{r}\right)^{\frac{1}{r}} d \mu^{N}(z) \\
& =\sqrt{2}^{M-1} \int_{\mathbb{T}^{N}}\left(\sum_{i_{M}=1}^{N}\left\|\sum_{\mathbf{j} \in \mathcal{M}(M-1, N)} v a_{\mathbf{j}, i_{M}} z_{j_{1}} \cdots z_{j_{M-1}}\right\|_{Y}^{r}\right)^{\frac{1}{r}} d \mu^{N}(z) .
\end{aligned}
$$


Finally we bound the integrand. We first apply that $v$ is $(r, 1)$-summing and in the final step [17, Theorem 1]

$$
\begin{aligned}
\left(\sum_{i_{M}=1}^{N} \|\right. & \left.\sum_{\mathbf{j} \in \mathcal{M}(M-1, N)} v a_{\mathbf{j}, i_{M}} z_{j_{1}} \cdots z_{j_{M-1}} \|_{Y}^{r}\right)^{\frac{1}{r}} \\
\leq & \pi_{r, 1}(v) \sup _{x^{\prime} \in B_{X^{\prime}}} \sum_{i_{M}=1}^{N}\left|x^{\prime}\left(\sum_{\mathbf{j} \in \mathcal{M}(M-1, N)} a_{\mathbf{j}, i_{M}} z_{j_{1}} \cdots z_{j_{M-1}}\right)\right| \\
& =\pi_{r, 1}(v) \sup _{x^{\prime} \in B_{X^{\prime}}} \sup _{y \in B_{\ell_{\infty}^{N}}}\left|\sum_{i_{M}=1}^{N} x^{\prime}\left(\sum_{\mathbf{j} \in \mathcal{M}(M-1, N)} a_{\mathbf{j}, i_{M}} z_{j_{1}} \cdots z_{j_{M-1}} y_{i_{M}}\right)\right| \\
& =\pi_{r, 1}(v) \sup _{x^{\prime} \in B_{X^{\prime}}} \sup _{y \in B_{\ell_{\infty}^{N}}}\left|x^{\prime}(A(z, \ldots, z, y))\right| \\
& \leq \pi_{r, 1}(v)\left(1+\frac{1}{M-1}\right)^{M-1} \sup _{x^{\prime} \in B_{X^{\prime}}}\left\|x^{\prime} \circ P\right\|_{\infty}=\pi_{r, 1}(v)\left(1+\frac{1}{M-1}\right)^{M-1}\|P\|_{\infty} .
\end{aligned}
$$

This gives the conclusion.

Proof of Theorem 4.2. In order to keep the notation as simple as possible we define

$$
\omega_{M}:=\frac{(q-1) M-q\left(\frac{1}{r}-\frac{1}{q}\right)}{q M} .
$$

Given $N$ and an $M$-homogeneous Dirichlet series $\sum a_{n} n^{-s}$, we define the $M$-homogeneous polynomial

$$
P: \ell_{\infty}^{N} \rightarrow X, \quad P(z)=\sum_{\mathbf{j} \in \mathcal{J}(M, N)} b_{\mathbf{j}} z_{j_{1}} \cdots z_{j_{M}}
$$

where

$$
b_{\mathbf{j}}=b_{j_{1} \ldots j_{M}}:= \begin{cases}a_{p_{j_{1}} \cdots p_{j_{M}}} & \text { if } p_{j_{1}} \cdots p_{j_{M}} \leq N . \\ 0 & \text { else. }\end{cases}
$$

We begin by splitting the sum and then bounding it using Hölder's inequality with $q$ and $q^{*}$ :

$$
\begin{aligned}
\sum_{n=1}^{N} \frac{\left\|v a_{n}\right\|_{Y}}{n^{\omega_{M}}} & (\log n)^{\lambda}=\sum_{\mathbf{j} \in \mathcal{J}(M, N)} \frac{\left\|v b_{\mathbf{j}}\right\|_{Y}}{\left(p_{j_{1}} \cdots p_{j_{M}}\right)^{\omega_{M}}}\left(\log p_{j_{1}} \cdots p_{j_{M}}\right)^{\lambda} \\
& \leq \sum_{j_{M}=1}^{N} \frac{\left(M \log p_{j_{M}}\right)^{\lambda}}{p_{j_{M}} \omega_{M}} \sum_{\substack{j_{1}, \ldots, j_{M-1} \\
j_{1} \leq \ldots \leq j_{M}}} \frac{\left\|v b_{\mathbf{j}}\right\|_{Y}}{\left(p_{j_{1}} \cdots p_{j_{M-1}}\right)^{\omega_{M}}} \\
& \leq M^{\lambda} \sum_{j_{M}=1}^{N} \frac{\left(\log p_{j_{M}}\right)^{\lambda}}{p_{j_{M}} \omega_{M}}\left(\sum_{\substack{j_{1}, \ldots, j_{M-1}: \\
j_{1} \leq \ldots \leq j_{M}}}\left\|v b_{\mathbf{j}}\right\|_{Y}^{q}\right)^{\frac{1}{q}}\left(\sum_{\substack{j_{1}, \ldots, j_{M-1}: \\
j_{1} \leq \ldots \leq j_{M}}}\left(p_{j_{1}} \cdots p_{j_{M-1}}\right)^{-q^{*} \omega_{M}}\right)^{\frac{1}{q^{*}}} \\
& \leq M^{\lambda} \sum_{j_{M}=1}^{N} \frac{\left(\log p_{j_{M}}\right)^{\lambda}}{p_{j_{M}} \omega_{M}}\left(\sum_{\substack{\sum_{j_{1}, \ldots, j_{M-1}:}: \\
j_{1} \leq \ldots \leq j_{M}}}\left\|v b_{\mathbf{j}}\right\|_{Y}^{q}\right)^{\frac{1}{q}}\left(\sum_{\substack{j_{M}-1 \\
q_{M}}} p_{j}^{-q^{*} \omega_{M}}\right)^{\frac{q^{*}}{2}} .
\end{aligned}
$$


Using the fact that for $0<\alpha<1$

$$
\sum_{p \leq x} p^{-\alpha} \underset{\sim}{\sim} \frac{x^{1-\alpha}}{\log x}
$$

(see e.g. [26, Satz 4.2, p.22] we get

$$
\left(\sum_{j \leq j_{M}} p_{j}{ }^{-q^{*} \omega_{M}}\right)^{\frac{M-1}{q^{*}}} \ll\left(\frac{p_{j_{M}}{ }^{1-q^{*} \omega_{M}}}{\log p_{j_{M}}}\right)^{\frac{M-1}{q^{*}}} .
$$

Using this and again Hölder's inequality (with $r$ and $r^{*}$ ) we finally obtain the following bound:

$$
\begin{aligned}
\sum_{n=1}^{N} \frac{\left\|v a_{n}\right\|_{Y}}{n^{\omega_{M}}}(\log n)^{\lambda} & \ll M^{\lambda} \sum_{j_{M}=1}^{N} \frac{\left(\log p_{j_{M}}\right)^{\lambda-\frac{M-1}{q^{*}}}}{p_{j_{M}} \frac{r-1}{r}}\left(\sum_{\substack{j_{1}, \ldots, j_{M-1} \\
j_{1} \leq \ldots \leq \leq j_{M}}}\left\|v b_{\mathbf{j}}\right\|_{Y}^{q}\right)^{\frac{1}{q}} \\
& \leq M^{\lambda}\left(\sum_{j_{M}=1}^{N} \frac{\left(\log p_{j_{M}}\right)^{r^{*}\left(\lambda-\frac{M-1}{q^{*}}\right)}}{p_{j_{M}}}\right)^{\frac{1}{r^{*}}}\left(\sum_{j_{M}=1}^{N}\left(\sum_{\substack{j_{1}, \ldots, j_{M-1} \\
j_{1} \leq \ldots \leq j_{M}}}\left\|v b_{\mathbf{j}}\right\|_{Y}^{q}\right)^{\frac{r}{q}}\right)^{\frac{1}{r}} .
\end{aligned}
$$

The left factor in the upper product converges for all $\lambda<\frac{M-1}{q^{*}}=\frac{q-1}{q}(M-1)$ since, by the prime number theorem, we have

$$
\left(\sum_{j_{M}=1}^{N} \frac{\left(\log p_{j_{M}}\right)^{r^{*}\left(\lambda-\frac{M-1}{q^{*}}\right)}}{p_{j_{M}}}\right)^{\frac{1}{r^{*}}} \ll\left(\sum_{n=1}^{N} \frac{(\log (n \log n))^{r^{*}\left(\lambda-\frac{M-1}{q^{*}}\right)}}{n \log n}\right)^{\frac{1}{r^{*}}} .
$$

For the right factor Lemma 4.3 and $(2.2)$ finally give

$$
\left(\sum_{j_{M}=1}^{N}\left(\sum_{\substack{j_{1}, \ldots, j_{M-1} \\ j_{1} \leq \ldots \leq j_{M}}}\left\|v b_{\mathbf{j}}\right\|_{Y}^{q}\right)^{\frac{r}{q}}\right)^{\frac{1}{r}} \ll \sup _{z \in B_{\ell_{\infty}^{N}}}\left\|\sum_{\mathbf{j} \in \mathcal{J}(M, N)} b_{\mathbf{j}} z_{j_{1}} \cdots z_{j_{M}}\right\|_{X}=\sup _{t \in \mathbb{R}}\left\|\sum_{n=1}^{N} a_{n} n^{-i t}\right\|_{X},
$$

and this gives the conclusion.

\section{LOWER ESTIMATES}

Getting lower estimates requires to find $M$-homogeneous Dirichlet series satisfying certain properties. We find them following the ideas of Maurizi and Queffélec in the scalar case (see 25, Theorem 3.1]). In one case (Theorem 5.1) we use probabilistic techniques, whereas in the other case (Theorem 5.4 we give a deterministic way through Schur matrices.

Theorem 5.1. Fix $1 \leq p \leq 2$ and $M, K \in \mathbb{N}$. Then for every $N$ there exists an $M$ homogeneous Dirichlet polynomial $\sum_{n=1}^{N} c_{n} n^{-s}$ in $\ell_{p}^{K}$ such that for every $p<q$ we have (with constants not depending on $N$ )

$$
\sum_{n=1}^{N}\left\|c_{n}\right\|_{q} \sim K^{\frac{1}{q}} \frac{N}{(\log N)^{M}}
$$

and

$$
\sup _{t \in \mathbb{R}}\left\|\sum_{n=1}^{N} c_{n} n^{-i t}\right\|_{p} \ll \frac{N^{\frac{1}{2}}}{(\log N)^{\frac{M}{2}}} K^{\frac{1}{p}} .
$$


For the proof we need first two lemmas.

Lemma 5.2. Given $M$ there exists a constant $C>0$ such that for every finite $I \subset\{n \in \mathbb{N} \mid$ $\Omega(n)=M\}$ and every choice of scalars $\left(a_{n}\right)_{n \in I} \subset \mathbb{C}$ we have

$$
\begin{aligned}
\int \| \sum_{n \in I} a_{n} g_{n}(\omega) z_{1}^{\alpha_{1}(n)} \cdots z_{r}^{\alpha_{r}(n)} & \|_{\mathcal{P}\left(M \ell_{\infty}^{r}, \mathbb{C}\right)} d \omega \\
& \leq C\left[\max _{n \in I}\left|a_{n}\right| \sqrt{1+\log \operatorname{card} I}+\left(\sum_{n \in I}\left|a_{n}\right|^{2}\right)^{\frac{1}{2}} \sqrt{r \log M}\right],
\end{aligned}
$$

where $g_{n}$ for $n \in I$ are independent Gaussian random variables and $r=\max \{\pi(n) \mid n \in I\}$.

Proof. We choose independent Gaussian random variables $\left(g_{n}\right)_{n \in I}$ and consider for each $\omega$ the $M$-homogeneous polynomial

$$
P_{\omega}(z)=\sum_{n \in I} a_{n} g_{n}(\omega) z_{1}^{\alpha_{1}(n)} \cdots z_{r}^{\alpha_{r}(n)}
$$

Then [19, Chapter 6, Theorem 3] implies that there is a constant $C_{1}>0$ such that

$$
\mathfrak{P}\left(\left\|P_{\omega}\right\|_{\infty} \geq C_{1}\left(\sum_{n \in I}\left|a_{n}\right|^{2}\right)^{\frac{1}{2}} \sqrt{r \log M}\right) \leq \frac{1}{M^{2} e^{r}}
$$

Moreover, by [22, Proposition 6.8] we have

$$
\int\left\|P_{\omega}\right\|_{\infty} d \omega \leq 6 \int \max _{n \in I}\left\|a_{n} g_{n}(\omega) z_{1}^{\alpha_{1}(n)} \cdots z_{r}^{\alpha_{r}(n)}\right\|_{\infty} d \omega+6 t_{0}
$$

where

$$
t_{0}=\inf \left\{t>0 \mid \mathfrak{P}\left(\left\|P_{\omega}\right\|_{\infty}>t\right) \leq \frac{1}{8,3}\right\}
$$

Now, (5.3) and the fact that $M^{2} e^{r} \geq 8,3$ for $M, r \geq 2$, give that $t_{0} \leq C_{2}\left(\sum_{n=1}^{N}\left|a_{n}\right|^{2}\right)^{\frac{1}{2}} \sqrt{r \log M}$ for some $C_{2}>0$. On the other hand,

$$
\begin{array}{r}
\int \max _{n \in I}|| a_{n} g_{n}(\omega) z_{1}^{\alpha_{1}(n)} \cdots z_{r}^{\alpha_{r}(n)} \|_{\infty}=\int \max _{n \in I}\left|a_{n} g_{n}(\omega)\right| \sup _{z \in B_{\ell_{\infty}^{r}}}\left|z_{1}^{\alpha_{1}(n)} \cdots z_{r}^{\alpha_{r}(n)}\right| d \omega \\
\leq \max _{n \in I}\left|a_{n}\right| \int \max _{n \in I}\left|g_{n}(\omega)\right| d \omega \ll\left(\max _{n \in I}\left|a_{n}\right|\right) \sqrt{1+\log \operatorname{card} I},
\end{array}
$$

where the last inequality follows from [28, Proposition 45.1]. This completes the proof.

Lemma 5.3. Given $M \in \mathbb{N}$ there exists a constant $C>0$ such that for every $1 \leq p \leq 2$, every $K \in \mathbb{N}$, every finite $I \subset\{n \in \mathbb{N} \mid \Omega(n)=M\}$ and every choice of scalars $\left(a_{n}\right)_{n \in I}$ in $\mathbb{C}$ 
we have

$$
\begin{aligned}
& \int\left\|\sum_{n \in I} a_{n}\left(\sum_{k=1}^{K} \varepsilon_{k n}(\omega) e_{k}\right) z_{1}^{\alpha_{1}(n)} \cdots z_{r}^{\alpha_{r}(n)}\right\|_{\mathcal{P}\left(M \ell_{\infty}^{r}, \ell_{p}^{K}\right)} d \omega \\
& \leq C\left[\left(\max _{n \in I}\left|a_{n}\right| \sqrt{1+\log \operatorname{card} I}+\left(\sum_{n \in I}\left|a_{n}\right|^{2}\right)^{\frac{1}{2}} \sqrt{r \log M}\right) K^{\frac{1}{p}-\frac{1}{2}}\right. \\
&\left.\quad+\max _{n \in I}\left(\left|a_{n}\right| \sqrt{\frac{\alpha(n) !}{M !}}\right) r^{\frac{M}{2}} K^{\frac{1}{p}}\right],
\end{aligned}
$$

where $\left(\varepsilon_{n k}\right)_{n, k}$ is a family of Rademacher random variables and $r=\max \{\pi(n) \mid n \in I\}$.

Proof. We choose independent Gaussian random variables $g_{n k}$ for $n \in I$ and $k=1, \ldots, K$. It is a well known fact that the Rademacher averages are dominated by the Gaussian averages (see [16, Proposition 12.11]):

$$
\begin{gathered}
\int\left\|\sum_{n \in I} a_{n}\left(\sum_{k=1}^{K} \varepsilon_{k n}(\omega) e_{k}\right) z_{1}^{\alpha_{1}(n)} \cdots z_{r}^{\alpha_{r}(n)}\right\|_{\mathcal{P}_{M}\left(\ell_{\infty}^{r}, \ell_{p}^{K}\right)} d \omega \\
\ll \int\left\|\sum_{n \in I} a_{n}\left(\sum_{k=1}^{K} g_{k n}(\omega) e_{k}\right) z_{1}^{\alpha_{1}(n)} \cdots z_{r}^{\alpha_{r}(n)}\right\|_{\mathcal{P}_{M}\left(\ell_{\infty}^{r}, \ell_{p}^{K}\right)} d \omega \\
=\int\left\|\sum_{\substack{n \in I \\
1 \leq k \leq K}} g_{k n}(\omega)\left(a_{n} z_{1}^{\alpha_{1}(n)} \cdots z_{r}^{\alpha_{r}(n)} \otimes e_{k}\right)\right\|_{\mathcal{P}\left({ }^{M} \ell_{\infty}^{r}\right) \otimes_{\varepsilon} \ell_{p}^{K}} d \omega .
\end{gathered}
$$

By Chevet's inequality [28, Corollary 3.2] we have

$$
\begin{aligned}
\int\left\|\sum_{\substack{n \in I \\
1 \leq k \leq K}} g_{k n}(\omega)\left(a_{n} z_{1}^{\alpha_{1}(n)} \cdots z_{r}^{\alpha_{r}(n)} \otimes e_{k}\right)\right\|_{\mathcal{P}\left({ }^{M} \ell_{\infty}^{r}\right) \otimes_{\varepsilon} \ell_{p}^{K}} d \omega \\
\ll\left[\sup _{\substack{y^{\prime} \in \mathcal{P}\left(\mathcal{R}^{M} \ell_{\infty}^{r}\right)^{\prime} \\
\left\|y^{\prime}\right\| \leq 1}}\left(\sum_{n \in I}\left|y^{\prime}\left(a_{n} z_{1}^{\alpha_{1}(n)} \cdots z_{r}^{\alpha_{r}(n)}\right)\right|^{2}\right)^{\frac{1}{2}} \int\left\|\sum_{k=1}^{K} g_{k}(\omega) e_{k}\right\|_{\ell_{p}^{K}} d \omega\right. \\
\left.\quad+\sup _{y^{\prime} \in B_{\ell_{p}^{K}}}\left(\sum_{k=1}^{K}\left|y^{\prime}\left(e_{k}\right)\right|^{2}\right)^{\frac{1}{2}} \int\left\|\sum_{n \in I} g_{n}(\omega) z_{1}^{\alpha_{1}(n)} \cdots z_{r}^{\alpha_{r}(n)}\right\|_{\mathcal{P}\left({ }^{M} \ell_{\infty}^{r}\right)} d \omega\right]
\end{aligned}
$$

Note that for $1 \leq p \leq 2$

$$
\sup _{y^{\prime} \in B_{\ell_{p}^{K^{\prime}}}}\left(\sum_{k=1}^{K}\left|y^{\prime}\left(e_{k}\right)\right|^{2}\right)^{\frac{1}{2}}=\sup _{x \in B_{\ell_{p^{\prime}}}}\left(\sum_{k=1}^{K}\left|x_{k}\right|^{2}\right)^{\frac{1}{2}}=\| \text { id }: \ell_{p^{\prime}}^{K} \rightarrow \ell_{2}^{K} \|=K^{\frac{1}{p}-\frac{1}{2}}
$$


and from the proof of 15 , Lemma 4.2] we know that

$$
\begin{aligned}
\sup _{\substack{y^{\prime} \in \mathcal{P}\left(\mathcal{P}^{M} \ell_{\infty}^{r}\right)^{\prime} \\
\left\|y^{\prime}\right\| \leq 1}}\left(\sum_{n \in I}\left|y^{\prime}\left(a_{n} z^{\alpha(n)}\right)\right|^{2}\right)^{\frac{1}{2}} & \leq \sup _{n \in I}\left(\left|a_{n}\right| \sqrt{\frac{\alpha(n) !}{M !}}\right) \| \text { id }: \ell_{\infty}^{r} \rightarrow \ell_{2}^{r} \|^{M} \\
& =\sup _{n \in I}\left(\left|a_{n}\right| \sqrt{\frac{\alpha(n) !}{M !}}\right) r^{\frac{M}{2}}
\end{aligned}
$$

Moreover, by [28, Proposition 45.1]

$$
\int\left\|\sum_{k=1}^{K} g_{k}(\omega) e_{k}\right\|_{\ell_{p}^{K}} d \omega \ll K^{\frac{1}{p}}
$$

Finally, Lemma 5.2 gives the conclusion.

Proof of Theorem 5.1. We fix $N$ and $M$ and consider

$$
r=\pi\left(N^{\frac{1}{M}}\right) .
$$

We define the set $I=\left\{n \in \mathbb{N} \mid n=p_{i_{1}} \cdots p_{i_{M}}, 1 \leq i_{1}<i_{2} \ldots<i_{M} \leq r\right\}$. Note that by the definition of $r$ for each $n \in I$ we have $n \leq p_{r}^{M} \leq N$. Given $K \in \mathbb{N}$ and Rademacher random variables $\left(\varepsilon_{n k}\right)_{\substack{1 \leq n \leq N \\ 1 \leq k \leq K}}$, we define the $M$-homogeneous Dirichlet polynomial $D_{\omega}$ in $\ell_{p}^{K}$ by

$$
D_{\omega}(s)=\sum_{n=1}^{N} c_{n} n^{-s}:=\sum_{n=1}^{N} a_{n}\left(\sum_{k=1}^{K} \varepsilon_{n k}(\omega) e_{k}\right) n^{-s},
$$

where

$$
a_{n}= \begin{cases}1 & \text { if } n \in I \\ 0 & \text { if } n \notin I\end{cases}
$$

Then we have

$$
\left\|c_{n}\right\|_{q}=\left(\sum_{k=1}^{K}\left|a_{n} \varepsilon_{n k} e_{k}\right|^{q}\right)^{\frac{1}{q}}=\left|a_{n}\right| K^{\frac{1}{q}}
$$

and hence

$$
\sum_{n=1}^{N}\left\|c_{n}\right\|_{q}=K^{\frac{1}{q}} \operatorname{card} I
$$

By the prime number theorem $r=\pi\left(N^{\frac{1}{M}}\right) \sim \frac{N \frac{1}{M}}{\log N}$, and hence $\operatorname{card} I=\left(\begin{array}{c}r \\ M\end{array}\right) \sim \frac{r^{M}}{M !} \sim \frac{N}{(\log N)^{M}}$. This gives 5.1.

We take now the $M$-homogeneous polynomial $P_{\omega}=\mathfrak{B}^{-1}\left(D_{\omega}\right)$. By Lemma 5.3 and 2.2 we 
have

$$
\begin{aligned}
& \int\left\|D_{\omega}\right\|_{\infty} d \omega=\int\left\|P_{\omega}\right\|_{\infty} d \omega \\
& \ll\left[\left(\max _{n \in I}\left|a_{n}\right| \sqrt{1+\log \operatorname{card} I}+\left(\sum_{n \in I}\left|a_{n}\right|^{2}\right)^{\frac{1}{2}} \sqrt{r \log M}\right) K^{\frac{1}{p}-\frac{1}{2}}\right. \\
&\left.\quad+\sup _{n \in I}\left(\left|a_{n}\right| \sqrt{\frac{\alpha(n) !}{M !}}\right) r^{\frac{M}{2}} K^{\frac{1}{p}}\right]
\end{aligned}
$$

Using again the estimate for card $I$ and the fact that $\sup _{\alpha \in \Lambda(M, r)} \sqrt{\frac{\alpha !}{M !}} \leq 1$ we easily get that the second summand is the one that increases faster. This gives $(5.2)$ and completes the proof.

Our second lower estimate will follow from the following result.

Theorem 5.4. Let $1 \leq p \leq 2$. Then for each $K$ there exists an $M$-homogeneous polynomial $P: \ell_{\infty}^{M K} \rightarrow \ell_{p}^{K}, P(z)=\sum_{|\alpha|=M} c_{\alpha} z^{\alpha}$, such that

$$
\sum_{\alpha \in \Lambda(M, M K)}\left\|c_{\alpha}\right\|_{2}=K^{M}
$$

and

$$
\sup _{z \in B_{\ell=\infty}^{M K}}\left\|\sum_{\alpha \in \Lambda(M, M K)} c_{\alpha} z^{\alpha}\right\|_{p} \leq K^{\frac{M}{2}+\frac{1}{p}-\frac{1}{2}}
$$

Proof. Let $\left(a_{i j}\right)_{i, j}$ be a complex $K \times K$-matrix satisfying

$$
\sum_{k=1}^{K} a_{k l} \bar{a}_{k m}=K \delta_{l m} \quad \text { and } \quad\left|a_{l m}\right|=1 \quad \text { for all } l, m \in \mathbb{N}
$$

(for example take $a_{m l}=e^{\frac{2 \pi i m l}{K}}$ ). With this we define the $M$-homogeneous polynomial $P$ : $\ell_{\infty}^{M K} \rightarrow \ell_{p}^{K}$ by

$$
P(z)=\sum_{\mathbf{i} \in \mathcal{M}(M, M K)} a_{1 i_{M}} a_{i_{M} i_{M-1}} \cdots a_{i_{2} i_{1}} z_{i_{1}} z_{K+i_{2}} \cdots z_{(M-1) K+i_{M}} e_{i_{M}}
$$


Let us show that $P$ satisfies (5.5). Indeed, if $z \in B_{\ell_{\infty}^{M K}}$, we have by the conditions of the matrix $\left(a_{i j}\right)_{i, j}$ that

$$
\begin{aligned}
\|P(z)\|_{2}^{2}= & \sum_{i_{M}=1}^{K}\left|\sum_{\mathbf{i} \in \mathcal{M}(M-1, K)} a_{1 i_{M}} a_{i_{M} i_{M-1}} \cdots a_{i_{2} i_{1}} z_{i_{1}} \cdots z_{(M-1) K+i_{M}}\right|^{2} \\
\leq & \sum_{i_{M}=1}^{K}\left|\sum_{\mathbf{i} \in \mathcal{M}(M-1, K)} a_{i_{M} i_{M-1}} \cdots a_{i_{2} i_{1}} z_{i_{1}} \cdots z_{(M-2) K+i_{M-1}}\right|^{2} \\
= & \sum_{i_{M}=1}^{K} \sum_{\mathbf{i}, \mathbf{j} \in \mathcal{M}(M-1, K)} a_{i_{M} i_{M-1}} \bar{a}_{i_{M} j_{M-1}} \cdots z_{(M-2) K+i_{M-1}} \bar{z}_{(M-2) K+j_{M-1}} \\
= & \left(\sum_{\mathbf{i}, \mathbf{j} \in \mathcal{M}(M-1, K)} a_{i_{M-1} i_{M-2}} \bar{a}_{j_{M-1} j_{M-2}} \cdots z_{(M-2) K+i_{M-1}} \bar{z}_{(M-2) K+j_{M-1}}\right. \\
& \left.\cdot \sum_{i_{M}=1}^{K} a_{i_{M} i_{M-1}} \bar{a}_{i_{M} j_{M-1}}\right) \\
= & \left.K \sum_{i_{M-1}=1}^{K}\left|z_{(M-2) K+i_{M-1}}\right|^{2} \sum_{\mathbf{i}, \mathbf{j} \in \mathcal{M}(M-2, K)} a_{i_{M-1} i_{M-2}} \cdots z_{(M-3) K+i_{M-2}}\right|^{2} \\
\leq & K \sum_{i_{M-1}=1}^{K}\left|\sum_{\mathbf{i}, \mathbf{j} \in \mathcal{M}(M-2, K)} a_{i_{M-1} i_{M-2}} \cdots a_{i_{2} i_{1}} z_{i_{1}} \cdots z_{(M-3) K+i_{M-2}}\right|^{2} .
\end{aligned}
$$

Repeating this argument we finally end up in

$$
\begin{aligned}
\|P(z)\|_{2}^{2} \leq K^{M-2} \sum_{i_{2}=1}^{K}\left|\sum_{i_{1}=1}^{K} a_{i_{2} i_{1}} z_{i_{1}}\right|^{2}=K^{M-2} \sum_{i_{1}, j_{1}=1}^{K}\left(\sum_{i_{2}=1}^{K} a_{i_{2} i_{1}} \bar{a}_{i_{2} j_{1}}\right) z_{i_{1}} \bar{z}_{j_{1}} \\
=K^{M-1} \sum_{i_{1}=1}^{K}\left|z_{i_{1}}\right|^{2} \leq K^{M} .
\end{aligned}
$$

Thus,

$$
\sup _{z \in B_{\ell_{\infty}^{K M}}}\|P(z)\|_{p} \leq\left\|\mathrm{id}: \ell_{p}^{K} \hookrightarrow \ell_{2}^{K}\right\| \sup _{z \in B_{\ell_{\infty}^{K M}}}\|P(z)\|_{2} \leq K^{\frac{1}{p}-\frac{1}{2}} K^{\frac{M}{2}} .
$$

On the other hand $P$ satisfies (5.4) since

$$
\begin{aligned}
\sum_{\alpha \in \Lambda(M, M K)}\left\|c_{\alpha}\right\|_{2} & =\sum_{\mathbf{i} \in \mathcal{M}(M, K)}\left\|a_{1 i_{M}} a_{i_{M} i_{M-1}} \cdots a_{i_{2} i_{1}} e_{i_{M}}\right\|_{2} \\
& =\sum_{\mathbf{i} \in \mathcal{M}(M, K)}\left(\sum_{k=1}^{K}\left|a_{1 i_{M}} a_{i_{M} i_{M-1}} \cdots a_{i_{2} i_{1}} e_{i_{M}}(k)\right|^{2}\right)^{\frac{1}{2}}=\sum_{\mathbf{i} \in \mathcal{M}(M, K)} 1=K^{M} .
\end{aligned}
$$




\section{ESTIMATES FOR OPERATORS IN $\ell_{p}$ SPACES}

We begin with an estimate of $Q_{N}(v)$ when $v$ is the inclusion from some $\ell_{p}$ to some other $\ell_{q}$. This gives a sort of quantified version of (1.8) and (1.9).

Theorem 6.1. Let $1 \leq p<q \leq \infty$. Then with constants depending only on $p, q$ we have:

$$
Q_{N}\left(\text { id }: \ell_{p} \hookrightarrow \ell_{q}\right) \leq \begin{cases}\frac{\sqrt{N}}{e^{\left(\sqrt{\frac{1}{p}-\max \left\{\frac{1}{2}, \frac{1}{q}\right\}}+o(1)\right) \sqrt{\log N \log \log N}}} & \text { if } 1 \leq p<2 \\ N^{1-\frac{1}{p}} & \text { if } p \geq 2 .\end{cases}
$$

For $1 \leq p \leq 2$ and $\lambda<\frac{M-1}{2}$ we have

$$
\frac{N^{\frac{M-2\left(\frac{1}{p}-\max \left\{\frac{1}{q}, \frac{1}{2}\right\}\right)}{2 M}}}{(\log N)^{\frac{M-2\left(\frac{1}{p}-\max \left\{\frac{1}{q}, \frac{1}{2}\right\}\right)}{2}}} \ll Q_{N}^{M}\left(\mathrm{id}: \ell_{p} \hookrightarrow \ell_{q}\right) \ll \frac{N^{\frac{M-2\left(\frac{1}{p}-\max \left\{\frac{1}{q}, \frac{1}{2}\right\}\right)}{2 M}}}{(\log N)^{\lambda}},
$$

and for $2 \leq p<q<\infty$ and $\lambda<\frac{q-1}{q}(M-1)$

$$
Q_{N}^{M}\left(\mathrm{id}: \ell_{p} \hookrightarrow \ell_{q}\right) \ll \frac{N^{1-\frac{1}{p}}}{(\log N)^{\lambda}} .
$$

Proof. The estimate in (6.1) follows immediately from Theorem 4.1 and the Bennett-Carl inequalities (see Section 2); indeed if $1 \leq p<q \leq 2$, then $\ell_{q}$ has cotype $2=2$-concave and the inclusion $\ell_{p} \hookrightarrow \ell_{q}$ is $(r, 1)$-summing with $\frac{1}{r}=\frac{1}{2}+\frac{1}{p}-\frac{1}{q}$. If $1 \leq p<2 \leq q$, we clearly have that $Q_{N}\left(\ell_{p} \hookrightarrow \ell_{q}\right) \leq Q_{N}\left(\ell_{p} \hookrightarrow \ell_{2}\right)$ and hence this case follows from the preceding one. Finally, for $2 \leq p$ we have $Q_{N}\left(\ell_{p} \hookrightarrow \ell_{q}\right) \leq Q_{N}\left(\ell_{p} \hookrightarrow \ell_{p}\right)$ and the estimate is a consequence of Remark 1 .

The upper estimates in (6.2) and (6.3) follow easily from Theorem 4.2. For $1 \leq p \leq q \leq 2$ the space $\ell_{q}$ is 2-concave and the Bennett-Carl inequalities (see Section 2 imply that the embedding $\ell_{p} \hookrightarrow \ell_{q}$ is $(r, 1)$-summing for $\frac{1}{r}=\frac{1}{2}+\frac{1}{p}-\frac{1}{q}$. Then Theorem 4.2 implies that for every $M$-homogeneous Dirichlet series $\sum a_{n} n^{-s}$ and $\lambda<\frac{M-1}{2}$ we have

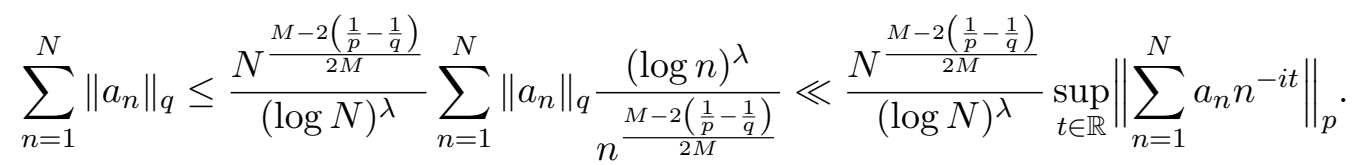

If $q \geq 2$ we clearly have that $Q_{N}^{M}\left(\ell_{p} \hookrightarrow \ell_{q}\right) \leq Q_{N}^{M}\left(\ell_{p} \hookrightarrow \ell_{2}\right)$. The case $2 \leq p$ follows in the same way.

For the lower estimate in (6.2) let us first note that the case $1 \leq p \leq 2<q$ follows immediately from the case $q=2$ since clearly $Q_{N}^{M}$ (id : $\left.\ell_{p} \hookrightarrow \ell_{2}\right) \leq Q_{N}^{M}$ (id : $\ell_{p} \hookrightarrow \ell_{q}$ ). We choose $K$ to be the biggest natural number smaller than $\frac{\pi^{\left(N^{\frac{1}{M}}\right)}}{M}$ and take the $M$-homogeneous polynomial $\sum c_{\alpha} z^{\alpha}$ from Theorem 5.4. Then for every $\alpha \in \Lambda(M, M K)$ we have that $p^{\alpha} \leq$ $p_{M K}^{M} \leq N$. We define the $M$-homogeneous Dirichlet-polynomial $\sum_{n=1}^{N} a_{n} n^{-s}$ by

$$
a_{p^{\alpha}}= \begin{cases}c_{\alpha} & \text { if } \alpha \in \Lambda(M, M K) \\ 0 & \text { else }\end{cases}
$$


Clearly,

$$
\sum_{n=1}^{N}\left\|a_{n}\right\|_{2}=\sum_{\alpha \in \Lambda(M, M K)}\left\|c_{\alpha}\right\|_{2}=K^{M}
$$

and by $(2.2)$

$$
\sup _{t \in \mathbb{R}}\left\|\sum_{n=1}^{N} a_{n} n^{i t}\right\|_{p}=\sup _{z \in B_{\ell_{\infty}^{M K}}}\left\|_{\alpha \in \Lambda(M, M K)} c_{\alpha} z^{\alpha}\right\|_{p} \leq K^{\frac{M}{2}+\frac{1}{p}-\frac{1}{2}}
$$

Hence

$$
Q_{N}^{M}\left(\mathrm{id}: \ell_{p} \hookrightarrow \ell_{2}\right) \geq K^{\frac{M}{2}-\left(\frac{1}{p}-\frac{1}{2}\right)}
$$

By the definition of $K$ and the prime number theorem $K \gg \frac{N^{\frac{1}{M}}}{M \log \left(N^{\frac{1}{M}}\right)}=\frac{N^{\frac{1}{M}}}{\log N}$, i.e.

$$
Q_{N}^{M}\left(\mathrm{id}: \ell_{p} \hookrightarrow \ell_{2}\right) \gg\left(\frac{N^{\frac{1}{M}}}{\log N}\right)^{\frac{M-2\left(\frac{1}{p}-\frac{1}{2}\right)}{2}}
$$

In this way we get a lower estimate for the case $q=2$ using a deterministic argument. This case follows also by a probabilistic argument, that is actually more reaching, since it also gives the lower estimate for $1 \leq p<q \leq 2$. Given $N$ and $M$ we take $K=\pi\left(N^{\frac{1}{M}}\right)$ in Theorem 5.1 . This gives

$$
\frac{N^{1+\frac{1}{M q}}}{(\log N)^{M+\frac{1}{q}}} \ll Q_{N}^{M}\left(\mathrm{id}: \ell_{q} \hookrightarrow \ell_{p}\right) \frac{N^{\frac{1}{2}+\frac{1}{M p}}}{(\log N)^{\frac{M}{2}+\frac{1}{p}}}
$$

and this gives the conclusion.

Finally, the quantified version of 1.10 is

Theorem 6.2. Given an $v: \ell_{1} \rightarrow \ell_{q}$, we have

$$
Q_{N}(v) \ll \begin{cases}\frac{\sqrt{N}}{e^{\left(\frac{1}{\sqrt{2}}+o(1)\right) \sqrt{\log N \log \log N}}} & \text { for } 1<q \leq 2 \\ N^{\frac{1}{2}+\varepsilon} & \text { for } 2 \leq q<\infty\end{cases}
$$

and

$$
Q_{N}^{M}(v) \ll \begin{cases}\frac{N^{\frac{1}{2}-\frac{1}{M}\left(1-\frac{1}{q}\right)}}{(\log N)^{\lambda}} & \text { for } 1 \leq q \leq 2 \text { and } 0<\lambda<\frac{M-1}{2} \\ \frac{N^{\left(1-\frac{1}{q}\right)-\frac{1}{2 M}}}{(\log N)^{\lambda}} & \text { for } 2 \leq q \leq \frac{2 M}{M-1} \text { and } 0<\lambda<\frac{q-1}{q}(M-1) \\ N^{\frac{1}{2}+\varepsilon} & \text { for } \frac{2 M}{M-1}<q<\infty .\end{cases}
$$

Proof. Let us note first that for $v: \ell_{1} \rightarrow \ell_{q}$ we can always do

$$
\sum_{n=1}^{N}\left\|v a_{n}\right\|_{q} \leq\|v\| \sum_{n=1}^{N}\left\|a_{n}\right\|_{1} \leq\|v\| Q_{N}\left(\ell_{1}\right) \sup _{t \in \mathbb{R}}\left\|\sum_{n=1}^{N} a_{n} n^{i t}\right\|_{1} .
$$

Then (3.3) gives that both $Q_{N}(v)$ and $Q_{N}^{M}(v)$ are $\ll N^{\frac{1}{2}+\varepsilon}$. If $1 \leq q \leq 2$, then $\ell_{q}$ is 2-concave and, by Kwapień's theorem (see Section 2 ) every operator $v: \ell_{1} \rightarrow \ell_{q}$ is $(r, 1)$-summing for 
$\frac{1}{r}=\frac{3}{2}-\frac{1}{q}$. On the other hand $\ell_{q}$ for $2 \leq q<\infty$ is $q$-concave and every operator is $(r, 1)$ summing for $\frac{1}{r}=\frac{1}{2}+\frac{1}{q}$. These facts, together with Theorem 4.1 and Theorem 4.2 give the conclusion. Note that for $\frac{2 M}{M-1}<q<\infty$ we have $\left(1-\frac{1}{q}\right)-\frac{1}{2 M}>\frac{1}{2}$.

\section{The WidTh of the STRIPs ARE ATtained}

By the very definition of the number $S(v)$ of an operator $v: X \rightarrow Y$ we have that for every Dirchlet series in $\mathcal{H}^{\infty}(X)$,

$$
\sum_{n=1}^{\infty}\left\|v a_{n}\right\|_{Y} \frac{1}{n^{S(v)+\varepsilon}}<\infty .
$$

The following vector valued analog of (1.1) (and also (1.5)) shows that in certain situations this inequality even holds for $\varepsilon=0$, and that we can even go a little bit further.

Proposition 7.1. Let $Y$ be a q-concave Banach lattice, with $2 \leq q<\infty$, and $v: X \rightarrow Y$ an $(r, 1)$-summing operator with $1 \leq r<q$.

(1) For every $D=\sum a_{n} n^{-s} \in \mathcal{H}^{\infty}(X)$ and every $\varepsilon>0$,

$$
\sum_{n=1}^{\infty}\left\|v a_{n}\right\|_{Y} \frac{e^{\left(2 \frac{q-1}{q} \sqrt{\frac{1}{r}-\frac{1}{q}}-\varepsilon\right) \sqrt{\log n \log \log n}}}{n^{1-\frac{1}{q}}}<\infty .
$$

(2) For every $D=\sum a_{n} n^{-s} \in \mathcal{H}_{M}^{\infty}(X)$ and every $0<\lambda<\frac{q-1}{q}(M-1)$

$$
\sum_{n=1}^{\infty}\left\|v a_{n}\right\|_{Y} \frac{(\log n)^{\lambda}}{n^{\frac{(q-1) M-q\left(\frac{1}{r}-\frac{1}{q}\right)}{q M}}}<\infty .
$$

Proof. For the proof of (1) let us note first that combining [1, Theorem 1.1] with the HahnBanach Theorem we have that there is a constant $C>0$ such that for every Dirichlet series in $\mathcal{H}^{\infty}(X)$

$$
\left\|\sum_{n=1}^{N} a_{n} n^{-s}\right\| \leq C \log N\left\|\sum_{n=1}^{\infty} a_{n} n^{-s}\right\| .
$$

Let $\tau:=2 \frac{q-1}{q} \sqrt{\frac{1}{r}-\frac{1}{q}}$. Since the sequence $e^{(\tau-\varepsilon) \sqrt{\log n \log \log n}} n^{\frac{1}{q}-1}$ is decreasing from some $n_{0}$ on, we have, using Theorem 4.1 .

$$
\begin{aligned}
\sum_{n=1}^{\infty} \frac{\left\|v a_{n}\right\|_{Y}}{n^{\frac{q-1}{q}}} & e^{(\tau-\varepsilon) \sqrt{\log n \log \log n}} \ll \sum_{k=0}^{\infty} \frac{e^{(\tau-\varepsilon) \sqrt{\log 2^{k} \log \log 2^{k}}}}{2^{\frac{q-1}{q}}} \sum_{n=2^{k}}^{k+1}\left\|v a_{n}\right\|_{Y} \\
& \ll \sum_{k=0}^{\infty} \frac{e^{(\tau-\varepsilon) \sqrt{\log 2^{k} \log \log 2^{k}}}}{e^{(\tau+o(1)) \sqrt{\log 2^{k+1} \log \log 2^{k+1}}}} \log 2^{k+1}\|D\|_{\infty} \ll\|D\|_{\infty} \sum_{k=0}^{\infty} \frac{k+1}{e^{\frac{\varepsilon}{2} \sqrt{k \log k}}}<\infty .
\end{aligned}
$$

On the other hand (2) follows from Theorem 4.1 proceeding as in [1, page 297].

Clearly this result applies to the embeddings id $: \ell_{p} \hookrightarrow \ell_{q}$ and for operators $v: \ell_{1} \rightarrow \ell_{q}$.

\section{ACKNOWLEDGEMENTS}

We would like to thank the referee for her/his comments and suggestions that helped improving the final shape of the paper. 


\section{REFERENCES}

[1] R. Balasubramanian, B. Calado, and H. Queffélec. The Bohr inequality for ordinary Dirichlet series. Studia Math., 175(3):285-304, 2006.

[2] F. Bayart. Hardy spaces of Dirichlet series and their composition operators. Monatsh. Math., 136(3):203236, 2002.

[3] G. Bennett. Inclusion mappings between $l^{p}$ spaces. J. Functional Analysis, 13:20-27, 1973.

[4] H. F. Bohnenblust and E. Hille. On the absolute convergence of Dirichlet series. Ann. of Math. (2), 32(3):600-622, 1931.

[5] H. Bohr. Über die Bedeutung der Potenzreihen unendlich vieler Variabeln in der Theorie der Dirichletschen Reihen $\sum \frac{a_{n}}{n^{s}}$. Nachr. Ges. Wiss. Göttingen, Math. Phys. Kl., pages 441-488, 1913.

[6] H. Bohr. Über die gleichmäßige Konvergenz Dirichletscher Reihen. J. Reine Angew. Math., 143:203-211, 1913.

[7] B. Carl. Absolut- $(p, 1)$-summierende identische Operatoren von $l_{u}$ in $l_{v}$. Math. Nachr., 63:353-360, 1974.

[8] F. Carlson. Contributions à la théorie des séries de Dirichlet. Note i. Ark. fö"r Mat., Astron. och Fys., 16(18):1-19, 1922.

[9] R. de la Bretèche. Sur l'ordre de grandeur des polynômes de Dirichlet. Acta Arith., 134(2):141-148, 2008.

[10] A. Defant, L. Frerick, J. Ortega-Cerdà, M. Ounaïes, and K. Seip. The Bohnenblust-Hille inequality for homogeneous polynomials is hypercontractive. Ann. of Math. (2), 174(1):485-497, 2011.

[11] A. Defant, D. García, M. Maestre, and D. Pérez-García. Bohr's strip for vector valued Dirichlet series. Math. Ann., 342(3):533-555, 2008.

[12] A. Defant, D. García, M. Maestre, and P. Sevilla-Peris. Bohr's strips for Dirichlet series in Banach spaces. Funct. Approx. Comment. Math., 44(part 2):165-189, 2011.

[13] A. Defant, M. Maestre, and U. Schwarting. Bohr radii of vector valued holomorphic functions. Adv. Math., 231(5):2837-2857, 2012.

[14] A. Defant, D. Popa, and U. Schwarting. Coordinatewise multiple summing operators in Banach spaces. J. Funct. Anal., 259(1):220-242, 2010.

[15] A. Defant and P. Sevilla-Peris. Convergence of Dirichlet polynomials in Banach spaces. Trans. Amer. Math. Soc., 363(2):681-697, 2011.

[16] J. Diestel, H. Jarchow, and A. Tonge. Absolutely summing operators, volume 43 of Cambridge Studies in Advanced Mathematics. Cambridge University Press, Cambridge, 1995.

[17] L. A. Harris. Bounds on the derivatives of holomorphic functions of vectors. In Analyse fonctionnelle et applications (Comptes Rendus Colloq. Analyse, Inst. Mat., Univ. Federal Rio de Janeiro, Rio de Janeiro, 1972), pages 145-163. Actualités Aci. Indust., No. 1367. Hermann, Paris, 1975.

[18] H. Hedenmalm, P. Lindqvist, and K. Seip. A Hilbert space of Dirichlet series and systems of dilated functions in $L^{2}(0,1)$. Duke Math. J., 86(1):1-37, 1997.

[19] J.-P. Kahane. Some random series of functions, volume 5 of Cambridge Studies in Advanced Mathematics. Cambridge University Press, Cambridge, second edition, 1985.

[20] S. V. Konyagin and H. Queffélec. The translation $\frac{1}{2}$ in the theory of Dirichlet series. Real Anal. Exchange, 27(1):155-175, 2001/02.

[21] S. Kwapień. Some remarks on $(p, q)$-absolutely summing operators in $l_{p}$-spaces. Studia Math., 29:327-337, 1968.

[22] M. Ledoux and M. Talagrand. Probability in Banach spaces. Classics in Mathematics. Springer-Verlag, Berlin, 2011. Isoperimetry and processes, Reprint of the 1991 edition.

[23] J. Lindenstrauss and L. Tzafriri. Classical Banach spaces. I. Springer-Verlag, Berlin, 1977. Sequence spaces, Ergebnisse der Mathematik und ihrer Grenzgebiete, Vol. 92.

[24] J. Lindenstrauss and L. Tzafriri. Classical Banach spaces. II, volume 97 of Ergebnisse der Mathematik und ihrer Grenzgebiete [Results in Mathematics and Related Areas]. Springer-Verlag, Berlin, 1979. Function spaces.

[25] B. Maurizi and H. Queffélec. Some remarks on the algebra of bounded Dirichlet series. J. Fourier Anal Appl, DOI 10.1007/s00041-009-9112-y, 2010.

[26] K. Prachar. Primzahlverteilung. Springer-Verlag, Berlin-Gttingen-Heidelberg, 1957.

[27] H. Queffélec. H. Bohr's vision of ordinary Dirichlet series; old and new results. J. Anal., 3:43-60, 1995. 
[28] N. Tomczak-Jaegermann. Banach-Mazur distances and finite-dimensional operator ideals, volume 38 of Pitman Monographs and Surveys in Pure and Applied Mathematics. Longman Scientific \& Technical, Harlow, 1989.

Institut für Mathematik. Universität Oldenburg. D-26111 Oldenburg (Germany)

E-mail address: andreas.defant@uni-oldenburg.de

E-mail address: ursula.schwarting@uni-oldenburg.de

Instituto Universitario de Matemática Pura y Aplicada and DMA, Etsiamn, Universitat Politècnica de València, CMno Vera s/n, 46022, Valencia, Spain

E-mail address: psevilla@mat.upv.es 